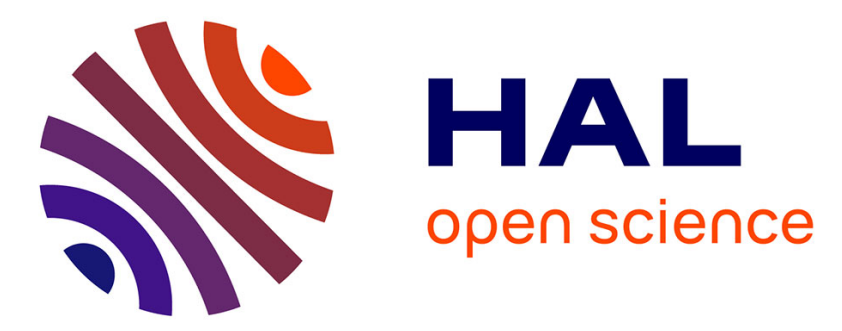

\title{
On the Well-Posedness, Stability And Accuracy Of An Asymptotic Model For Thin Periodic Interfaces In Electromagnetic Scattering Problems
}

\author{
Bérangère Delourme, Houssem Haddar, Patrick Joly
}

\section{- To cite this version:}

Bérangère Delourme, Houssem Haddar, Patrick Joly. On the Well-Posedness, Stability And Accuracy Of An Asymptotic Model For Thin Periodic Interfaces In Electromagnetic Scattering Problems. Mathematical Models and Methods in Applied Sciences, 2013. hal-00682357

\section{HAL Id: hal-00682357 https://hal.inria.fr/hal-00682357}

Submitted on 26 Mar 2012

HAL is a multi-disciplinary open access archive for the deposit and dissemination of scientific research documents, whether they are published or not. The documents may come from teaching and research institutions in France or abroad, or from public or private research centers.
L'archive ouverte pluridisciplinaire HAL, est destinée au dépôt et à la diffusion de documents scientifiques de niveau recherche, publiés ou non, émanant des établissements d'enseignement et de recherche français ou étrangers, des laboratoires publics ou privés. 


\title{
On the Well-Posedness, Stability And Accuracy Of An Asymptotic Model For Thin Periodic Interfaces In Electromagnetic Scattering Problems
}

\author{
Bérangère Delourme, Houssem Haddar, Patrick Joly
}

$01 / 2012$

\begin{abstract}
We analyze the well-posedness and stability properties of a parameter dependent problem that models the reflection and transmission of electromagnetic waves at a thin and rapidly oscillating interface. The latter is modeled using approximate interface conditions that can be derived using asymptotic expansion of the exact solution with respect to the small parameter (proportional to the periodicity length of oscillations and the width of the interface). The obtained uniform stability results are then used to analyze the accuracy (with respect to the small parameter) of the proposed model.
\end{abstract}

\section{Introduction}

This work is concerned with the analysis of an asymptotic model for the scattering of electromagnetic waves across thin interfaces at a fixed frequency. The considered model can be seen as a second order approximation of scattering from thin and rapidly (periodically) oscillating layers. The latter configuration appears in number of applications such as the scattering of electromagnetic waves from the ground (radar applications), the scattering of electromagnetic waves from the skin (simulation of cell phone radiations, microwave imaging), non destructive testing of coated dielectrics, etc... The simplified model configuration (motivated by a confidential industrial application) that we shall be interested in consists in studying the reflection and transmission of electromagnetic waves scattering from a plane, thin, and periodic layer: such a layer is made of an array of regularly spaced dielectric inclusions. The thickness of the layer and the distance between two consecutive obstacles are of the same order $\delta>0$, which is assumed to be much smaller than the wavelength of the incident wave. It is clear that direct numerical computations of such a problem becomes prohibitively expensive as the small parameter $\delta$ goes to 0 . The use of an asymptotic model, where the thin periodic layer is replaced by an approximate transmission condition, presents an interesting solution since the numerical discretization of this approximate problem is no longer constrained by the small scale and therefore is much cheaper. These approximate models can be derived from an asymptotic expansion of the solution of the exact problem with respect to the small parameter $\delta$. This is what has been done in Ref. [17] for acoustic waves and in Ref. [16] for electromagnetic waves. The present paper can 
be seen as a natural continuation of [17]. For the analysis and numerical simulation of the electromagnetic time dependent problem and for interfaces with constant coefficients we refer to Ref. [10].

More precisely, we shall mainly analyze in this paper the well-posedness and uniform stability (with respect to the small parameter $\delta$ ) of a family of transmission problems which are non standard in two ways. First they involve jumps across two very close but different surfaces (4). Second, they involve approximate transmission conditions (ATC's) depending on $\delta$ (5). Such conditions include of course the ones issued from the asymptotic analysis of thin and rough interfaces as it will be more clearly outlined in section 1.2. As a consequence of the stability analysis, we also will be able to rigously analyse the accuracy of the approximate transmission conditions. From this point of view, this paper can be viewed as a one step further the analysis of effective boundary conditions (also called generalized impedance boundary conditions or GIBC's) for rough surfaces, which has been the subject of a more abundant literature (see Ref. [3],[1],[37],[28],[21],[32],[20],[6]). Similar ATC's have been recently analyzed [17] in the case of the scalar wave equations (acoustic waves). As one can expect, the case of 3D Maxwell's equations that we consider here is more involved from the mathematical point of view.

\section{Outline of the paper and the main results.}

We first introduce the small parameter dependent problem $\mathcal{P}$ formed by equations (1), (2), (3) and (5) in section 1.1. The first main goal of this paper is to prove that this boundary value problem is well-posed (at least for $\delta$ small enough) and is stable uniformly with respect to the small parameter $\delta$. This is the object of sections 2 to 4 . We shall employ a variational approach and inf-sup conditions (Babuska's theory) that are established by compact perturbation techniques. The proof is much more complicated than in the scalar Helmholtz case, [17] since the compactness properties of the lower order terms in the variational formulation is much more difficult to obtain in the context of Maxwell's equations (see Ref. [36],[13], [14],[8] for more general investigations of this issue). The key point consists in building an appropriate Helmholtz decomposition of the solution that fits our transmission problem. This approach is classical in the analysis of Maxwell equations (see for instance Ref. [26],[23] and [29]). In section 2, after having rewritten the transmission conditions in an adequate form via the introduction of a boundary operator $\mathcal{G}_{\omega}$ (whose main properties are described in proposition 7), we establish the variational formulation of our transmission problem (see $(21,22,23,24)$ ) in an appropriate function framework (see $(25,27)$ ). In section 3 , we construct the appropriate (Helmholtz like) decomposition of the variational space (see section 3.1 for the main statements and section 3.2 for their proof). In particular, the uniform continuity properties of the related projection operators is established in proposition 9. Section 3.3 is devoted to the proof of the compactness results associated with this Helmholtz decomposition (Proposition 11). Section 4 is devoted to the proof of the well-posedness and stability result (theorem 16). The key point is the proof of uniform inf-sup conditions (proposition 18) that is achieved by using the results of section 3.

Let us remark that since our proofs are based on compactness and contradiction arguments, our well-posedness result is only proved for $\delta$ small enough (this is a standard restriction in the analysis of perturbation of non coercive problems), which is sufficient for the applications we have in mind. When considering the problem for a given (not 
necessarily small) $\delta$, the main hard issue with centered formulations of ATCs would be to ensure the uniqueness of solutions for all frequencies. One way to get around this difficulty would be to use a non symmetric (but consistent) version of the ATC (5) in which, contrary to (5), the two lips of the interface would not play a symmetric role. We refer the reader to Ref. [17] where this has been done for the scalar wave equation.

Our second main goal is to use the previous results to prove the accuracy of conditions (5) (using (9)) as effective transmission conditions for thin periodic interfaces as described in section 1.2. This is the subject of section 5 and more precisely proposition 19. The result appears as a consequence of the combination of the theoretical results from Ref. [16] and an asymptotic analysis with respect to $\delta$ of the transmission problem $\mathcal{P}$.

\section{Setting of the problem and outline of the paper}

\subsection{The model problem}

We are interested in the electromagnetic field $\mathbf{E}^{\delta}$ solution of time harmonic Maxwell's equations

$$
\operatorname{curl} \operatorname{curl} \mathbf{E}^{\delta}-\omega^{2} \mathbf{E}^{\delta}=f \text { in } \Omega_{\alpha \delta}^{+} \cup \Omega_{\alpha \delta}^{-},
$$

where $\omega$ denotes the pulsation of time variations, $f$ is a given source term in $L^{2}\left(\Omega_{\alpha \delta}^{+} \cup\right.$ $\left.\Omega_{\alpha \delta}^{-}\right)^{3}$ and the domains $\Omega_{\alpha \delta}^{ \pm}$are defined by (see Fig.1(a)):

$\Omega_{\alpha \delta}^{ \pm}:=\left\{\left(x_{1}, x_{2}, x_{3}\right) \in \mathbb{R}^{3},-\frac{L_{1}}{w}<x_{1}<L_{1} / 2,-\frac{L_{2}}{2}<x_{2}<\frac{L_{2}}{2}, \frac{\alpha \delta}{2}< \pm x_{3}<\frac{L_{3}}{2}\right\}$

where $L_{1}, L_{2}, L_{3}$ and $\alpha$ are given positive numbers and $\delta$ is a (small) positive parameter.

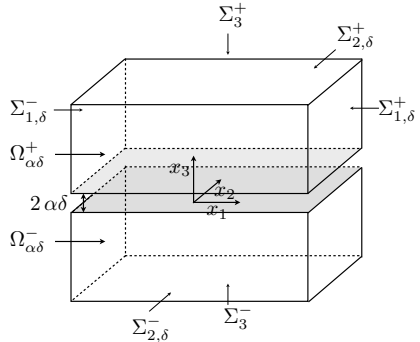

(a) Approximate configuration: domains $\Omega_{\alpha \delta}^{+}$and $\Omega_{\alpha \delta}^{-}$

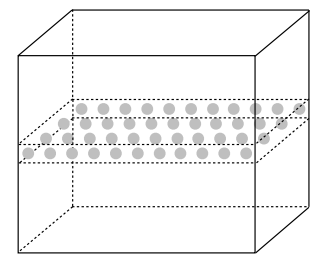

(b) "Exact" configuration: domain $\Omega$

Figure 1: Approximate and exact configurations

Let us denote by

$$
\Sigma_{i, \delta}^{ \pm}:=\left\{\left(x_{1}, x_{2}, x_{3}\right) \in \partial \Omega_{\alpha \delta}^{ \pm}, x_{i}= \pm L_{i} / 2\right\}
$$


for $i=1,2,3$ and set $\Sigma_{i}^{ \pm}:=\Sigma_{i, 0}^{ \pm}$. To simulate approximately a radiation condition in the $x_{3}$ direction we impose impedance boundary conditions of the form

$$
\pm e_{3} \times \operatorname{curl} \mathbf{E}^{\delta}=-i \omega \mathbf{E}_{T}^{\delta} \text { on } \Sigma_{3}^{ \pm}
$$

where we use the abbreviation $\mathbf{E}_{T}:=e_{3} \times\left(\mathbf{E} \times e_{3}\right)$ for the projection of $\mathbf{E}^{\delta}$ on the $\left(x_{1}, x_{2}\right)$ plane. This notation will be also adopted in the sequel. For lateral boundaries we impose periodicity conditions:

$$
\mathbf{E}^{\delta} \times\left. e_{i}\right|_{\Sigma_{i, \delta}^{+}}=\mathbf{E}^{\delta} \times\left. e_{i}\right|_{\Sigma_{i, \delta}^{-}}, \quad \text { and } \operatorname{curl} \mathbf{E}^{\delta} \times\left. e_{i}\right|_{\Sigma_{i, \delta}^{+}}=\operatorname{curl} \mathbf{E}^{\delta} \times\left. e_{i}\right|_{\Sigma_{i, \delta}^{-}}, i=1,2 .
$$

The problem will be completely set up by specifying the boundary conditions at $x_{3}=$ $\pm \alpha \delta$. We shall in fact impose transmission conditions that model the existence of a thin layer of width $\delta$ with material properties that may vary periodically in the lateral directions, with a periodicity length proportional to $\delta$. In order to present these transmission conditions we first need to introduce some notation. We shall denote

$$
\Gamma:=\left\{\left(x_{1}, x_{2}\right) \in \mathbb{R}^{2},-L_{1} / 2<x_{1}<L_{1} / 2, \quad-L_{2} / 2<x_{2}<L_{2} / 2,\right\}
$$

and sometimes abusively confuse it with $\partial \Omega_{0}^{+} \cap \partial \Omega_{0}^{-}$. For any sufficiently regular function or vector field $u$ defined on $\Omega_{\alpha \delta}^{-} \cap \Omega_{\alpha \delta}^{+}$we abbreviate

$$
u_{\alpha}^{ \pm}\left(x_{1}, x_{2}\right):=\lim _{x_{3} \rightarrow \pm \alpha \delta} u\left(x_{1}, x_{2}, x_{3}\right) ;\left(x_{1}, x_{2}\right) \in \Gamma
$$

and define the $\alpha$-jump and $\alpha$-mean value, $[u]_{\alpha}$ and $\langle u\rangle_{\alpha}$, by

$$
[u]_{\alpha}:=u_{\alpha}^{+}-u_{\alpha}^{-}, \quad\langle u\rangle_{\alpha}=\frac{1}{2}\left(u_{\alpha}^{+}+u_{\alpha}^{-}\right) \quad \text { on } \Gamma .
$$

The solutions $\mathbf{E}^{\delta}$ are required to satisfy the following transmission conditions

$$
\left\{\begin{array}{l}
(a) \quad\left[e_{3} \times \mathbf{E}^{\delta}\right]_{\alpha}+\delta\left(\frac{A_{e}}{\omega^{2}} \operatorname{curl}_{\Gamma} \operatorname{curl}_{\Gamma}\left\langle\left(\operatorname{curl} \mathbf{E}^{\delta}\right)_{T}\right\rangle_{\alpha}-\mathbf{D}_{e}\left\langle\left(\operatorname{curl} \mathbf{E}^{\delta}\right)_{T}\right\rangle_{\alpha}\right)=0 \\
(b) \quad\left[e_{3} \times \operatorname{curl} \mathbf{E}^{\delta}\right]_{\alpha}+\delta\left(A_{m} \operatorname{curl}_{\Gamma} \operatorname{curl}_{\Gamma}\left\langle\mathbf{E}_{T}^{\delta}\right\rangle_{\alpha}-\omega^{2} \mathbf{D}_{m}\left\langle\mathbf{E}_{T}^{\delta}\right\rangle_{\alpha}\right)=0
\end{array}\right.
$$

where, $A_{e}$ and $A_{m}$ are positive constants, $\mathbf{D}_{e}$ and $\mathbf{D}_{m}$ are some constant, positive definite and symmetric matrices that are independent of $\delta$. The tangential operators $\overrightarrow{c u r l}_{\Gamma}$ and $\operatorname{curl}_{\Gamma}$ are respectively defined by

$$
\operatorname{curl}_{\Gamma} u:=\left(\frac{\partial u}{\partial x_{2}},-\frac{\partial u}{\partial x_{1}}, 0\right)^{t} \quad \text { and } \quad \operatorname{curl}_{\Gamma} \mathbf{v}:=\frac{\partial \mathbf{v}_{2}}{\partial x_{1}}-\frac{\partial \mathbf{v}_{1}}{\partial x_{2}}
$$

where $u$ denotes a scalar function and $\mathbf{v}=\left(\mathbf{v}_{1}, \mathbf{v}_{2}\right)$ a tangential vector field on $\Gamma$. In the applications we have in mind, the quantities $A_{e}, A_{m}, \mathbf{D}_{e}$ and $\mathbf{D}_{m}$ are related to the material properties of the thin layer and may also depend on the parameter $\alpha$, as it will be explained in section 1.2. In what follows we shall denote $\mathcal{P}$ the boundary value problem defined by equations (1), (2), (3) and (5).

Remark 1 (On the the significance of the parameter $\alpha$ that defines the position of the two lips of the interface). As far as the problem $\mathcal{P}$ described in section 1.1 is concerned, we could have merely considered the case $\alpha=0$ which corresponds to the case where the two lips are sticked together. This would have allowed to avoid some (minor) 
technical difficulties that we had to treat (see the proofs) to cope with the fact that we deal with problems posed in domains that depend on $\delta$ (see the proofs of propositions 10 and 18). On the other hand, taking $\alpha$ into account becomes fundamental when we want to analyze the accuracy of effective transmission conditions for rough interfaces (sections 1.2 and 5).

Remark 2 (On the geometrical assumption and obvious generalizations). The considered simplified geometrical setting was motivated by the periodic transmission layer problem indicated in section 1.2. Let us however indicate that this setting as well as the simplified modeling of radiation conditions does not really affect the generality of our results, except those of section 5 dedicated to the asymptotic analysis of the problem of section 1.2. For instance all the results of sections 3 and 4 can be easily generalized to the case of $\Gamma$ being the boundary of a regular bounded domains. Also, modulo the use of tedious (but classical [29]) technical tools, one can also treat the case of locally non constant material properties and the case of unbounded domain with Sylver-Müller radiation condition at infinity.

\subsection{Motivation: the transmission problem for thin periodic layers}

Although we think that the analysis of transmission conditions of the form (5) presents its own interest from the mathematical point of view, the reason why we have chosen to study transmission conditions of the form (5) comes from the mathematical and numerical modeling of the reflection and transmission electromagnetic waves through thin highly heterogeneous interfaces. The transmission conditions have been constructed in Ref. [15], in the case of a flat interface (see also Ref. [15] for the case of a cylindrical interface). To be more precise, problem $\mathcal{P}$ corresponds to a first order approximate model associated with a thin periodic layer scattering problem. Let us describe the "exact" problem. First, we consider the domain

$$
\Omega:=\left\{\left(x_{1}, x_{2}, x_{3}\right) \in \mathbb{R}^{3},-L_{j} / 2<x_{j}<L_{j} / 2, \quad j=1,2,3\right\},
$$

and two periodic positive functions $\mu^{\delta}$ and $\epsilon^{\delta}$ (see Fig.1(b)) such that

$$
\epsilon^{\delta}:=\epsilon\left(\frac{x_{1}}{\delta}, \frac{x_{2}}{\delta}, \frac{x_{3}}{\delta}\right) \quad \text { and } \quad \mu^{\delta}:=\mu\left(\frac{x_{1}}{\delta}, \frac{x_{2}}{\delta}, \frac{x_{3}}{\delta}\right),
$$

where $\mu\left(\hat{x}_{1}, \hat{x}_{2}, \hat{x}_{3}\right)$ and $\epsilon\left(\hat{x}_{1}, \hat{x}_{2}, \hat{x}_{3}\right)$ are $L^{\infty}\left(\mathbb{R}^{3}\right)$ functions, 1-periodic in $\hat{x}_{1}$ and $\hat{x}_{2}$ and satisfying

$$
\left\{\begin{array} { l } 
{ 0 < \mu ^ { - } \leq \mu ( \hat { x } _ { 1 } , \hat { x } _ { 2 } , \hat { x } _ { 3 } ) \leq \mu ^ { + } , } \\
{ \mu ( \hat { x } _ { 1 } , \hat { x } _ { 2 } , \hat { x } _ { 3 } ) = 1 \text { if } | \hat { x } _ { 3 } | \geq \frac { 1 } { 2 } , }
\end{array} \quad \left\{\begin{array}{l}
0<\epsilon^{-} \leq \epsilon\left(\hat{x}_{1}, \hat{x}_{2}, \hat{x}_{3}\right) \leq \epsilon^{+}, \\
\epsilon\left(\hat{x}_{1}, \hat{x}_{2}, \hat{x}_{1}\right)=1 \text { if }\left|\hat{x}_{3}\right| \geq \frac{1}{2}
\end{array}\right.\right.
$$

Remark 3. We consider the same period in $\hat{x}_{1}$ and $\hat{x}_{2}$ only for simplicity. Considering two different periods would give completely similar results.

Moreover, we shall restrict ourselves to the case where the periodicity cell is symmetric (in the more general periodic case, one gets more complicated equivalent conditions)

$$
\mu \text { and } \epsilon \text { are even functions in } \hat{x}_{1} \text { and } \hat{x}_{2} .
$$

Given a (fixed) source term $f$ in $L^{2}(\Omega)^{3}$, the exact problem consists in finding $\mathbf{E}_{e}^{\delta}$ that satisfies Maxwell's equations

$$
\operatorname{curl}\left(\frac{1}{\mu^{\delta}} \operatorname{curl} \mathbf{E}_{e}^{\delta}\right)-\omega^{2} \epsilon^{\delta} \mathbf{E}_{e}^{\delta}=f \text { in } \mathcal{D}^{\prime}(\Omega),
$$


periodicity conditions (3), and the impedance condition (2). To link the "exact" problem to Problem $\mathcal{P}$, we need to introduce the periodicity cell

$$
\mathcal{B}:=\left\{\left(\hat{x}_{1}, x_{2}, \hat{x}_{3}\right) \in \mathbb{R}^{3}, \quad-1 / 2<\hat{x}_{1}<1 / 2,-1 / 2<\hat{x}_{2}<1 / 2\right\},
$$

and, for $i \in\{1,2,3\}, a=\epsilon$ or $a=\mu$, the so-called "profile" functions $p_{i}^{a}$ : these functions are the functions whose gradient is 1-periodic in $\hat{x}_{1} \hat{x}_{2}$, satisfy

$$
\operatorname{div}\left(a \nabla p_{i}^{a}\right)=0 \text { in } \mathcal{D}^{\prime}(\mathcal{B}),
$$

and have the following prescribed behaviors for large $\hat{x}_{3}$ : there exists a constant $C_{i} \in$ $\mathbb{R}^{3}$ and two functions $\left(g^{+}, g^{-}\right)$, that decrease exponentially as $\left|\hat{x}_{3}\right|$ goes to infinity, such that

$$
p_{i}^{a}= \pm C_{i}+\hat{x}_{i}+g^{ \pm} \quad \text { if } \pm \hat{x}_{3}>1 / 2,
$$

The existence and uniqueness of such profiles are proven in Ref. [17] and [15]. For $\alpha>0$, and $\mathcal{B}_{0}:=\mathcal{B} \cap\left\{\left|\hat{x}_{3}\right|<1 / 2\right\}$, we then define

$$
\begin{aligned}
& A_{e}:=2 \alpha-1+\int_{\mathcal{B}_{0}} \frac{\partial p_{3}^{\epsilon}}{\partial \hat{x}_{3}}, \\
& A_{m}:=2 \alpha-1+\int_{\mathcal{B}_{0}} \frac{\partial p_{3}^{\mu}}{\partial \hat{x}_{3}}, \\
& \mathbf{D}_{e}:=\left(\begin{array}{cc}
d_{1}^{e} & 0 \\
0 & d_{2}^{e}
\end{array}\right), \quad d_{j}^{e}=2 \alpha-1+\int_{\mathcal{B}_{0}} \mu \frac{\partial p_{j}^{\mu}}{\partial \hat{x}_{j}}, \quad j=1,2, \\
& \mathbf{D}_{m}:=\left(\begin{array}{cc}
d_{1}^{m} & 0 \\
0 & d_{2}^{m}
\end{array}\right), \quad d_{j}^{m}=2 \alpha-1+\int_{\mathcal{B}_{0}} \varepsilon \frac{\partial p_{j}^{\varepsilon}}{\partial \hat{x}_{j}}, \quad j=1,2 .
\end{aligned}
$$

In the end, we expect $\mathbf{E}^{\delta}$, the solution of problem $\mathcal{P}$ associated with (9), to be a good approximation of the exact solution $\mathbf{E}_{e}^{\delta}$. More precisely, we shall prove that, for $\left|x_{3}\right|$ large enough, $\mathbf{E}^{\delta}=\mathbf{E}_{e}^{\delta}+O\left(\delta^{2}\right)$ in a sense to be specified (see section 5). The reader will observe that all coefficients in (9) increase linearly with $\alpha$ and thus become positive as soon as $\alpha$ is large enough, satisfying the conditions announced in section 1.1. This will be exploited in section 5 .

\section{Variational formulation of the problem}

The jump condition (5-a) is not well adapted to variational formulations that involve a single unknown $\mathbf{E}^{\delta}$ : multiplying the equation curl curl $\mathbf{E}^{\delta}-\omega^{2} \mathbf{E}^{\delta}=f$ by a test function $\varphi$ and using the Stokes formulas one ends up with volumetric integrals and also boundary terms given by

$$
+\int_{\Gamma}\left\langle\left(\operatorname{curl} \mathbf{E}^{\delta}\right)_{T}\right\rangle_{\alpha} \cdot{\overline{\left[e_{3} \times \boldsymbol{\varphi}\right]_{\alpha}}}_{\alpha}-\int_{\Gamma}\left[e_{3} \times \operatorname{curl} \mathbf{E}^{\delta}\right]_{\alpha} \cdot \overline{\left\langle\boldsymbol{\varphi}_{T}\right\rangle_{\alpha}} .
$$

The second term can be easily treated by replacing $\left[e_{3} \times \operatorname{curl} \mathbf{E}^{\delta}\right]_{\alpha}$ with its expression given by (5-b). However, for the first term, we need to solve (5-a) as an equation for 
the quantity $\left\langle\left(\operatorname{curl} \mathbf{E}^{\delta}\right)_{T}\right\rangle_{\alpha}$ to express it in terms of $\left[e_{3} \times \mathbf{E}^{\delta}\right]_{\alpha}$. To do so, we shall need to exclude some exceptional frequencies $\omega$ (a discrete set) (see Hypothesis 6).

Remark 4. We conjecture that the restriction corresponding to Hypothesis 6 is not needed. It has been introduced essentially for technical reasons, in order to establish a variational formulation where the electric field is the only unknown. One of the reasons why we think this condition is artificial is that it gives a privileged role to $A_{e}$ and $\mathbf{D}_{e}$ with respect to $A_{m}$ and $\mathbf{D}_{m}$. However, the role of these coefficients would be interchanged if one would choose to work with the magnetic field, instead of the electric field, as unknown.

Let us introduce some notation for appropriate function spaces. With $H_{\#}^{s}(\Gamma)$ denoting the usual Sobolev spaces (by convention $H_{\#}^{0}=L^{2}$ ) of doubly periodic (with periods $L_{1}$ and $L_{2}$ ) functions on $\Gamma$, that are easily defined in terms of the Fourier coefficients of a periodic distribution $\varphi$ on $\Gamma$ :

$$
\widehat{\varphi}(\mathbf{k}):=\left\langle\varphi, e^{i \mathbf{k} \cdot \mathbf{x}}\right\rangle_{\Gamma}, \quad \mathbf{k}=\left(k_{1}, k_{2}\right) \in \frac{2 \pi}{L_{1}} \mathbb{Z} \times \frac{2 \pi}{L_{2}} \mathbb{Z},
$$

using the following characterization

$$
\varphi \in H_{\#}^{s}(\Gamma) \quad \Longleftrightarrow\|\varphi\|_{H_{\#}^{s}(\Gamma)}^{2}:=\sum_{\mathbf{k}}\left(1+|\mathbf{k}|^{2}\right)^{s}|\widehat{\varphi}(\mathbf{k})|^{2}<+\infty,
$$

we define

$$
H_{\#}^{s}\left(\operatorname{curl}_{\Gamma}, \Gamma\right):=\left\{u=\left(u_{1}, u_{2}, 0\right)^{t} \in H_{\#}^{s}(\Gamma)^{2} ; \operatorname{curl}_{\Gamma} u \in H_{\#}^{s}(\Gamma)\right\}
$$

equipped with the graph norm. Similarly define $H_{\#}^{s}\left(\operatorname{div}_{\Gamma}, \Gamma\right)$ by replacing curl $l_{\Gamma}$ with $\operatorname{div}_{\Gamma}$ where

$$
\operatorname{div}_{\Gamma} \mathbf{v}=\frac{\partial \mathbf{v}_{1}}{\partial x_{1}}+\frac{\partial \mathbf{v}_{2}}{\partial x_{2}} .
$$

The notation $H_{\#}\left(\operatorname{div}_{\Gamma}, \Gamma\right)\left(\right.$ resp. $\left.H_{\#}\left(\operatorname{curl}_{\Gamma}, \Gamma\right)\right)$ refers to $H_{\#}^{0}\left(\operatorname{div}_{\Gamma}, \Gamma\right)\left(\right.$ resp. $\left.H_{\#}^{0}\left(\operatorname{curl}_{\Gamma}, \Gamma\right)\right)$.

Given $g \in H_{\#}^{-1 / 2}\left(\operatorname{div}_{\Gamma}, \Gamma\right)$, we consider $u \in H_{\#}\left(\operatorname{curl}_{\Gamma}, \Gamma\right)$ solution to

$$
A_{e} \operatorname{curl}_{\Gamma} \operatorname{curl}_{\Gamma} u-\omega^{2} \mathbf{D}_{e} u=-g .
$$

Proposition 5. Given $g \in H_{\#}^{-1 / 2}\left(\operatorname{div}_{\Gamma}, \Gamma\right), \mathbf{D}_{e}$ a real $2 \times 2$ positive definite symmetric matrix and $A_{e}>0$, problem (13) has a unique solution $u \in H_{\#}\left(\operatorname{curl}_{\Gamma}, \Gamma\right)$ that depends continuously on g except for a discrete sequence of frequencies $\left(\omega_{n}\right)_{n \in \mathbb{N}}$ that admits $+\infty$ as only accumulation point.

One can use standard abstract arguments to prove this result, using an adapted 2D Helmholtz decomposition of vector fields and classical compactness arguments. Such a proof works for non constant (in space) $A_{e}$ and $\mathbf{D}_{e}$ modulo usual assumptions. In the "constant case" that we consider here, we can be more precise using Fourier series. One sees that each vector $\widehat{u}(k)$ (according to the notation (11)) satisfies

$$
R(\mathbf{k}) \widehat{u}(\mathbf{k})-\omega^{2} \mathbf{D}_{e} \widehat{u}(\mathbf{k})=-\widehat{g}(\mathbf{k})
$$

where $R(\mathbf{k})$ is the hermitian and positive operator in $\mathbb{C}^{2}$ defined by (with $\mathbf{k} \cdot v=$ $\mathbf{k}_{1} v_{1}+\mathbf{k}_{2} v_{2}$ )

$$
R(\mathbf{k}) v=|\mathbf{k}|^{2} v-(\mathbf{k} \cdot v) \mathbf{k}
$$


Therefore, the exceptional frequencies are the eigenvalues of the matrices $\mathbf{D}_{e}^{-1} R(\mathbf{k})$ which gives

$$
\left\{\omega_{n}, n \in \mathbb{N}\right\}=\{0\} \cap\left\{\lambda(\mathbf{k}), \quad \mathbf{k} \in \frac{2 \pi}{L_{1}} \mathbb{Z} \times \frac{2 \pi}{L_{2}} \mathbb{Z}\right\}
$$

where $\lambda(\mathbf{k}) \in \mathbb{R}_{+}^{*}$ is the unique non-zero eigenvalue of $\mathbf{D}_{e}^{-1} R(\mathbf{k})$. In the diagonal case (cf. (7)), we get

$$
\left\{\omega_{n}, n \in \mathbb{N}\right\}=\left\{\frac{d_{1}^{e} d_{2}^{e}}{\left(d_{1}^{e}\right)^{2}+\left(d_{2}^{e}\right)^{2}}\left(d_{1}^{e} k_{1}^{2}+d_{2}^{e} k_{2}^{2}\right), \quad \mathbf{k} \in \frac{2 \pi}{L_{1}} \mathbb{Z} \times \frac{2 \pi}{L_{2}} \mathbb{Z}\right\}
$$

These special frequencies referred to eigenfrequencies of problem (13) correspond to the values of $\omega$ for which there exist non trivial solutions of (13) for $g=0$. From now on we shall exclude these frequencies by making the assumption:

Hypothesis 6. The frequency $\omega$ is not an eigenfrequency of problem (13).

For $\omega$ satisfying assumption 6 we define the operator $\mathcal{G}_{\omega}$ by

$$
\begin{gathered}
\mathcal{G}_{\omega}: H_{\#}^{-1 / 2}\left(\operatorname{div}_{\Gamma}, \Gamma\right) \rightarrow H_{\#}\left(\operatorname{curl}_{\Gamma}, \Gamma\right) \\
g \mapsto \mathcal{G}_{\omega} g:=u
\end{gathered}
$$

where $u \in H_{\#}\left(\operatorname{curl}_{\Gamma}, \Gamma\right)$ is the unique solution of (13). In the sequel, we shall use extensively the following properties of the operator $\mathcal{G}_{\omega}$.

Proposition 7. The operator $\mathcal{G}_{\omega}$ is hermitian since, for any $\left(g, g^{\prime}\right) \in H^{-1 / 2}\left(\operatorname{div}_{\Gamma}, \Gamma\right)^{2}$,

$$
\left\langle\mathcal{G}_{\omega} g, g^{\prime}\right\rangle_{\Gamma}=-A_{e} \int_{\Gamma} \operatorname{curl}_{\Gamma} \mathcal{G}_{\omega} g \cdot \overline{\operatorname{curl}_{\Gamma} \mathcal{G}_{\omega} g^{\prime}} d s+\omega^{2} \int_{\Gamma} \mathbf{D}_{e} \mathcal{G}_{\omega} g \cdot \overline{\mathcal{G}_{\omega} g^{\prime}} d s
$$

and satisfies the following properties $\left(\mathbb{P}_{0}^{2}\right.$ denoting the space of constant tangential vector fields on $\Gamma$ )

$$
\begin{gathered}
\forall g \in H_{\#}^{-1 / 2}\left(\operatorname{div}_{\Gamma}, \Gamma\right), \quad \operatorname{div}_{\Gamma}\left(\mathbf{D}_{e}\left(\mathcal{G}_{\omega} g\right)\right)=\frac{1}{\omega^{2}} \operatorname{div}_{\Gamma} g . \\
\forall g \in H_{\#}^{-1 / 2}\left(\operatorname{div}_{\Gamma}, \Gamma\right), \quad \forall \mathbf{C} \in \mathbb{P}_{0}^{2}, \quad \int_{\Gamma} \mathbf{D}_{e}\left(\mathcal{G}_{\omega} g\right) \cdot \mathbf{C} d s=\frac{1}{\omega^{2}}\langle g, \mathbf{C}\rangle_{\Gamma}
\end{gathered}
$$

as well as the identity

$$
\forall g \in H_{\#}^{1}(\Gamma), \quad-A_{e} \int_{\Gamma} \operatorname{curl}_{\Gamma} \mathcal{G}_{\omega} g \overline{\operatorname{curl}_{\Gamma} g} d s+\omega^{2} \int_{\Gamma} \mathbf{D}_{e} \mathcal{G}_{\omega} g \cdot \bar{g} d s=\int_{\Gamma}|g|^{2} d s
$$

Finally, for all $g \in H^{-1 / 2}\left(\operatorname{div}_{\Gamma}, \Gamma\right), \mathcal{G}_{\omega}(g) \in H^{1 / 2}(\Gamma)$ and there exists a constant $C_{\omega}>0$ such that

$$
\begin{aligned}
& \text { (i) } \quad\left\|\mathcal{G}_{\omega}(g)\right\|_{H\left(\operatorname{curl}_{\Gamma}, \Gamma\right)} \leq C_{\omega}\|g\|_{H^{-1 / 2}\left(\operatorname{div}_{\Gamma}, \Gamma\right)}, \\
& \text { (ii) } \quad\left\|\operatorname{div}_{\Gamma}\left(\mathbf{D}_{e} \mathcal{G}_{\omega}(g)\right)\right\|_{H^{-1 / 2}(\Gamma)} \leq C_{\omega}\|g\|_{H^{-1 / 2}\left(\operatorname{div}_{\Gamma}, \Gamma\right)}, \\
& \text { (iii) } \quad\left\|\mathcal{G}_{\omega}(g)\right\|_{H^{1 / 2}(\Gamma)} \leq C_{\omega}\|g\|_{H^{-1 / 2}\left(\operatorname{div}_{\Gamma}, \Gamma\right)} .
\end{aligned}
$$


Proof. (14) and (17) are direct consequences of Green's formula. (15) is obtained by applying $\operatorname{div}_{\Gamma}$ to (13) and (16) is obtained by testing (13) against constant vector fields. The estimate (18)(i) is a direct consequence of the well-posedness of (13). The estimate (18)(ii) follows directly from (15). The last estimate is a consequence of the fact that if $u \in H\left(\operatorname{curl}_{\Gamma}, \Gamma\right)$ and $\operatorname{div}_{\Gamma}\left(\mathbf{D}_{e} \varphi\right) \in H^{-1 / 2}(\Gamma)$ then $u \in H^{1 / 2}(\Gamma)$. This follows, using Fourier transform, from the fact that there exists a constant $C>0$ such that

$$
\forall \mathbf{k} \in \frac{2 \pi}{L_{1}} \mathbb{Z} \times \frac{2 \pi}{L_{2}} \mathbb{Z}, \quad \forall v \in \mathbb{C}^{2}, \quad|\mathbf{k}|^{2}|v|^{2} \leq C\left(|\mathbf{k} \times v|^{2}+\left|\mathbf{k} \cdot \mathbf{D}_{e} v\right|^{2}\right)
$$

with $\mathbf{k} \times v=\mathbf{k}_{1} v_{2}-\mathbf{k}_{2} v_{1}$. To prove (19), it suffices to remark that, since $\mathbf{D}_{e}$ is positive definite, we have ( $\mathbf{S}$ and $\mathbb{S}$ denoting the unit spheres in $\mathbb{R}^{2}$ and $\mathbb{C}^{2}$ )

$$
\beta:=\inf _{(\theta, v) \in \mathbf{S} \times \mathbb{S}}\left(|\theta \times v|^{2}+\left|\theta \cdot \mathbf{D}_{e} v\right|^{2}\right)>0 .
$$

Indeed, if $\beta$ was equal to 0 , there would exist $(\theta, v) \in \mathbf{S} \times \mathbb{S}$ such that $\theta \times v=0$ and $\theta \cdot \mathbf{D}_{e} v=0$. Expanding $v$ as $v=v_{1} e_{1}+v_{2} e_{2}$ where $\left(e_{1}, e_{2}\right)$ is the orthonormal basis of eigenvectors of $\mathbf{D}_{e}$, the last two equations give a linear system in $\left(v_{1}, v_{2}\right)$ with determinant $\mathbf{D}_{e} \theta \cdot \theta \neq 0$. This implies $v=0$ which gives a contradiction. Then, it is immediate to see that (19) follows with $C=\beta^{-1}$.

Using the operator $\mathcal{G}_{\omega}$, the transmission condition (5-a) can be (formally) equivalently written as

$$
\left\langle\left(\operatorname{curl} \mathbf{E}^{\delta}\right)_{T}\right\rangle_{\alpha}=\frac{\omega^{2}}{\delta} \mathcal{G}_{\omega}\left(\left[n \times \mathbf{E}^{\delta}\right]_{\alpha}\right) .
$$

This expression can be used in the first term (10). One can check (after simple rearrangements) that a variational formulation of the problem defined by (1), (2), (2), (2-b) and (10) can be written in the standard form:

$$
\text { Find } \mathbf{E}^{\delta} \in X^{\delta} \text { such that } \forall \boldsymbol{\varphi} \in X^{\delta}, \quad a_{\delta}\left(\mathbf{E}^{\delta}, \boldsymbol{\varphi}\right)=\int_{\Omega_{\alpha \delta}^{ \pm}} f \cdot \bar{\varphi} d x
$$

where $X^{\delta}$ denotes the variational space specified in the sequel and where $a_{\delta}$ can be split into

$$
a_{\delta}(\boldsymbol{\psi}, \boldsymbol{\varphi}):=a_{\delta}^{+}(\boldsymbol{\psi}, \boldsymbol{\varphi})-b_{\delta}(\boldsymbol{\psi}, \boldsymbol{\varphi})
$$

with

$$
\begin{array}{r}
a_{\delta}^{+}(\boldsymbol{\psi}, \boldsymbol{\varphi}):=\int_{\Omega_{\alpha \delta}^{ \pm}} \operatorname{curl} \boldsymbol{\psi} \cdot \overline{\operatorname{curl} \boldsymbol{\varphi}} d x-i \omega \int_{\Sigma_{3}^{ \pm}} \boldsymbol{\psi}_{T} \cdot \overline{\boldsymbol{\varphi}} d s \\
-\delta A_{m} \int_{\Gamma} \operatorname{curl}_{\Gamma}\left\langle\boldsymbol{\psi}_{T}\right\rangle_{\alpha} \overline{\operatorname{curl}_{\Gamma}\left\langle\boldsymbol{\varphi}_{T}\right\rangle_{\alpha}} d s, \\
b_{\delta}(\boldsymbol{\psi}, \boldsymbol{\varphi}):=\int_{\Omega_{\alpha \delta}^{ \pm}} \omega^{2} \boldsymbol{\psi} \cdot \overline{\boldsymbol{\varphi}}_{T} d x+\delta \int_{\Gamma} \omega^{2} \mathbf{D}_{m}\left\langle\boldsymbol{\psi}_{T}\right\rangle_{\alpha} \cdot \overline{\left\langle\boldsymbol{\varphi}_{T}\right\rangle_{\alpha}} d s \\
-\frac{\omega^{2}}{\delta}\left\langle\mathcal{G}_{\omega}\left(\left[e_{3} \times \boldsymbol{\psi}\right]_{\alpha}\right), \overline{\left[e_{3} \times \boldsymbol{\varphi}\right]_{\alpha}}\right\rangle_{\Gamma},
\end{array}
$$


where $\langle\cdot, \cdot\rangle_{\Gamma}$ denotes a duality product between two function spaces defined on $\Gamma$ with pivot space $L^{2}(\Gamma)$ (the duality product coincides with the $L^{2}(\Gamma)$ scalar product for sufficiently regular functions). This notation will be used in the remaining of this paper. The splitting of $a_{\delta}$ has been chosen in the spirit of the Fredholm theory $: a_{\delta}^{+}$is a coercive bilinear form and $b_{\delta}$ will appear as a compact perturbation.

The expression of the variational form suggests to use as a variational space

$$
X^{\delta}:=\left\{\boldsymbol{\psi} \in H_{\#}\left(\operatorname{curl}, \Omega_{\alpha \delta}^{ \pm}\right),\left\langle\boldsymbol{\psi}_{T}\right\rangle_{\alpha} \in H_{\#}\left(\operatorname{curl}_{\Gamma}, \Gamma\right),\left.\boldsymbol{\psi}_{T}\right|_{\Sigma_{3}^{ \pm}} \in L^{2}\left(\Sigma_{3}^{ \pm}\right)^{3}\right\}
$$

where $H_{\#}\left(\operatorname{curl}, \Omega_{\alpha \delta}^{ \pm}\right):=\left\{\boldsymbol{\psi} \in H\left(\operatorname{curl}, \Omega_{\alpha \delta}^{ \pm}\right), \boldsymbol{\psi} \times\left. e_{i}\right|_{\Sigma_{i, \delta}^{+}}=\boldsymbol{\psi} \times\left. e_{i}\right|_{\Sigma_{i, \delta}^{-}}, i=1,2\right\}$.

$X^{\delta}$ is a Hilbert space when equipped with the natural graph norm

$$
u \mapsto\left(\|\boldsymbol{\psi}\|_{H\left(\operatorname{curl}, \Omega_{\alpha \delta}^{ \pm}\right)}^{2}+\left\|\langle\boldsymbol{\psi}\rangle_{\alpha}\right\|_{H\left(\operatorname{curl}_{\Gamma}, \Gamma\right)}^{2}+\left\|\boldsymbol{\psi}_{T}\right\|_{L^{2}\left(\Sigma_{3}^{ \pm}\right)}^{2}\right)^{\frac{1}{2}}
$$

However, it will be useful to work with another ( $\delta$-dependent) norm of $X^{\delta}$

$$
\begin{aligned}
\|\boldsymbol{\psi}\|_{X^{\delta}}^{2} & :=\|\boldsymbol{\psi}\|_{H\left(\operatorname{curl}, \Omega_{\alpha \delta}^{ \pm}\right)}^{2}+\left\|\boldsymbol{\psi}_{T}\right\|_{L^{2}\left(\Sigma_{3}^{ \pm}\right)}^{2}+\frac{1}{\delta}\left\|\mathcal{G}_{\omega}\left(\left[e_{3} \times \boldsymbol{\psi}\right]_{\alpha}\right)\right\|_{H\left(\operatorname{curl}_{\Gamma}, \Gamma\right)}^{2} \\
& +\delta\left\|\left\langle\boldsymbol{\psi}_{T}\right\rangle_{\alpha}\right\|_{H\left(\operatorname{curl}_{\Gamma}, \Gamma\right)}^{2}
\end{aligned}
$$

The choice of this norm is guided by the following expression of $a_{\delta}(\psi, \psi)$ that is obtained from (22) after using (14) in (24):

$$
\begin{aligned}
a_{\delta}(\boldsymbol{\psi}, \boldsymbol{\psi})= & \|\operatorname{curl} \boldsymbol{\psi}\|_{L^{2}\left(\Omega_{\alpha \delta}^{ \pm}\right)}^{2}-\omega^{2}\|\boldsymbol{\psi}\|_{L^{2}\left(\Omega_{\alpha \delta}^{ \pm}\right)}^{2}+A_{m} \delta\left\|\operatorname{curl}_{\Gamma}\left\langle\boldsymbol{\psi}_{T}\right\rangle_{\alpha}\right\|_{L^{2}(\Gamma)}^{2} \\
& -\delta \omega^{2} \int_{\Gamma} \mathbf{D}_{m}\left\langle\boldsymbol{\psi}_{T}\right\rangle_{\alpha} \cdot \overline{\left\langle\boldsymbol{\psi}_{T}\right\rangle_{\alpha}} d s \\
& +\frac{\omega^{4}}{\delta} \int_{\Gamma} \mathbf{D}_{e} \mathcal{G}_{\omega}\left(\left[e_{3} \times \boldsymbol{\psi}\right]_{\alpha}\right) \cdot \overline{\mathcal{G}_{\omega}\left(\left[e_{3} \times \boldsymbol{\psi}\right]_{\alpha}\right)} d s \\
& -\frac{A_{e} \omega^{2}}{\delta}\left\|\operatorname{curl}_{\Gamma} \mathcal{G}_{\omega}\left(\left[e_{3} \times \boldsymbol{\psi}\right]_{\alpha}\right)\right\|_{L^{2}(\Gamma)}^{2}-i \omega \int_{\Sigma_{3}^{ \pm}}\left|\boldsymbol{\psi}_{T}\right|^{2} d s .
\end{aligned}
$$

For fixed $\delta$, one observes thanks to Proposition 7 that the norm (27) is equivalent to the graph norm (26). We therefore deduce that $X^{\delta}$ equipped with (27) is a Hilbert space. Moreover, one easily checks, thanks to Proposition 7 and trace theorems for $H$ (curl) spaces [4], [9]), that $a_{\delta}^{+}$and $b_{\delta}$ are continuous on $X^{\delta} \times X^{\delta}$ with continuity constants independent of $\delta$ (which justifies the norm (27)).

$\forall(\boldsymbol{\psi}, \boldsymbol{\varphi}) \in X^{\delta} \times X^{\delta}, \quad a_{\delta}^{+}(\boldsymbol{\psi}, \boldsymbol{\varphi}) \leq C\|\boldsymbol{\psi}\|_{X^{\delta}}\|\boldsymbol{\varphi}\|_{X^{\delta}}, \quad b_{\delta}(\boldsymbol{\psi}, \boldsymbol{\varphi}) \leq C\|\boldsymbol{\psi}\|_{X^{\delta}}\|\boldsymbol{\varphi}\|_{X^{\delta}}$

Remark 8 (Notation convention). When present only in one side of a formula (equality or inequality), the short notation $\mathrm{O}^{ \pm}$refers to $\mathrm{O}^{+} \cup \mathrm{O}^{-}$for given domains $\mathrm{O}^{+}$and $O^{-}$. For instance in those cases $\int_{O^{ \pm}} \equiv \int_{O^{+} \cup O^{-}}$. However, when present in both sides of a formula, this notation refers to the usual meaning, i.e. the formula is valid for both cases + and - . 


\section{A Helmholtz decomposition of $X^{\delta}$}

As it is classical for Maxwell's equations, the lower order terms, represented here by $b_{\delta}$, are not a compact perturbation of the main operator, represented here by $a_{\delta}^{+}$, if the variational space does not include some control of the divergence of the fields and some boundary terms (cf. Ref AmroucheBernardiDaugeGirault,Proposition 2.7). This is the case for $X^{\delta}$ which is not compactly embedded into $L^{2}\left(\Omega_{\alpha \delta}^{ \pm}\right)^{3}$. A Helmholtz decomposition of the space $X^{\delta}$ is therefore needed (see Ref [19]). More precisely we shall decompose $X^{\delta}$ into the form $X_{0}^{\delta} \oplus \nabla S^{\delta}$ where $X_{0}^{\delta}$ is compactly embedded into $L^{2}\left(\Omega_{\alpha \delta}^{ \pm}\right)^{3}$ and write an equivalent variational formulation on $X_{0}^{\delta}$. This procedure is well known and inspired by the approach developed for instance in [26], [27] (see also Ref [29], Chapters 4 and 9). We find also this kind of technique in [31, 23].

The main difficulty is to find the decomposition that fits the structure of the variational problem (21). For instance if one considers the subspace of $X^{\delta}$ of divergence free functions, then this subspace is still not compactly embedded in $L^{2}\left(\Omega_{\alpha \delta}^{ \pm}\right)^{3}$. The one we suggest here is already indicated by the proposed splitting of $a_{\delta}$. More precisely we define $S^{\delta}$ by (modulo the obvious identification between $\Sigma_{i, \delta}^{+}$and $\Sigma_{i, \delta}^{-}$)

$S^{\delta}:=\left\{p \in H^{1}\left(\Omega_{\alpha \delta}^{ \pm}\right),\langle p\rangle_{\alpha} \in H^{1}(\Gamma), p=0\right.$ on $\left.\Sigma_{3}^{ \pm},\left.p\right|_{\Sigma_{i, \delta}^{+}}-\left.p\right|_{\Sigma_{i, \delta}^{-}} \in \mathbb{P}_{0}, i=1,2\right\}$,

equipped with the graph norm (note that Poincaré's inequality applies to functions in $\left.S^{\delta}\right)$

$$
\left(\|\nabla p\|_{L^{2}\left(\Omega_{\alpha \delta}^{ \pm}\right)}^{2}+\left\|\langle p\rangle_{\alpha}\right\|_{H^{1}(\Gamma)}^{2}\right)^{1 / 2} .
$$

One easily checks that $\nabla S^{\delta}:=\left\{\nabla p, p \in S^{\delta}\right\}$ is a closed subspace of $X^{\delta}$ and that $a_{\delta}^{+}$ vanishes on $\nabla S^{\delta}$ :

$$
\forall p \in S^{\delta}, \quad \forall \boldsymbol{\varphi} \in X^{\delta}, \quad a_{\delta}^{+}(\nabla p, \boldsymbol{\varphi})=a_{\delta}^{+}(\boldsymbol{\varphi}, \nabla p)=0 .
$$

We then define $X_{0}^{\delta}$ as

$$
X_{0}^{\delta}:=\left\{\boldsymbol{\psi} \in X^{\delta}, b_{\delta}(\boldsymbol{\psi}, \nabla p)=0, \forall p \in S^{\delta}\right\}
$$

and observe that, according to (32), we also have

$$
X_{0}^{\delta}=\left\{\boldsymbol{\psi} \in X^{\delta}, a_{\delta}(\psi, \nabla p)=0, \forall p \in S^{\delta}\right\} \text { (by using (32)). }
$$

Interpreting the variational equation $b_{\delta}(\boldsymbol{\psi}, \nabla p)=0, \forall p \in S^{\delta}$ in the distributional sense leads to the following equivalent definition of $X_{0}^{\delta}$.

$$
\begin{gathered}
X_{0}^{\delta}=\left\{\boldsymbol{\psi} \in X^{\delta}, \operatorname{div} \boldsymbol{\psi}=0 \text { in } \Omega_{\alpha \delta}^{ \pm},\left.\boldsymbol{\psi} \cdot e_{i}\right|_{\Sigma_{i, \delta}^{+}}=\left.\boldsymbol{\psi} \cdot e_{i}\right|_{\Sigma_{i, \delta}^{-}}, i=1,2,\right. \\
\left\langle\boldsymbol{\psi} \cdot e_{3}\right\rangle_{\alpha}=\frac{1}{\delta} \operatorname{curl}_{\Gamma} \mathcal{G}_{\omega}\left(\left[e_{3} \times \boldsymbol{\psi}\right]_{\alpha}\right) \\
\left.\quad \text { and }-\left[\boldsymbol{\psi} \cdot e_{3}\right]_{\alpha}=\delta \operatorname{div}_{\Gamma}\left[\mathbf{D}_{e}\left\langle\boldsymbol{\psi}_{T}\right\rangle_{\alpha}\right] \text { on } \Gamma\right\}
\end{gathered}
$$

The remaining of this section is dedicated to the proof of the following proposition.

Proposition 9 (A Helmholtz decomposition of $X^{\delta}$ ). Assume that Hypothesis 6 holds. Then, 
(i)- $X_{0}^{\delta}$ and $\nabla S^{\delta}$ are closed subspaces of $X^{\delta}$,

(ii)- For sufficiently small $\delta$, the space $X^{\delta}$ is the direct sum of $X_{0}^{\delta}$ and $\nabla S^{\delta}$. Furthermore, the projection operators associated with this sum are bounded (with a continuity modulus independent of $\delta$ ).

The proof of this proposition is postponed to subsection 3.2. We refer to the direct sum of point (ii) of this theorem by writing

$$
X^{\delta}=X_{0}^{\delta} \oplus \nabla S^{\delta}
$$

where the sign $\oplus$ refers also to orthogonality with respect to $b_{\delta}(\cdot, \cdot)$ (as this is obviously the case from the definition of $X_{0}^{\delta}$ ). We remark that if this decomposition holds then, for a given $\boldsymbol{\psi} \in X^{\delta}, \boldsymbol{\psi}=\nabla p+\boldsymbol{\psi}_{0}$ where $p \in S^{\delta}$ and $\boldsymbol{\psi}_{0} \in X_{0}^{\delta}$ satisfies $b_{\delta}\left(\boldsymbol{\psi}_{0}, \nabla q\right)=$ 0 for all $q \in S^{\delta}$. Therefore $p$ (and then $\psi_{0}$ ) can be constructed from $\psi$ by solving the variational problem

$$
b_{\delta}(\nabla p, \nabla q)=b_{\delta}(\boldsymbol{\psi}, \nabla q) \quad \forall q \in S^{\delta} .
$$

The first main step in the construction of the Helmholtz decomposition is then to prove that (36) is well posed. We shall assume in the sequel that Hypothesis 6 holds.

\subsection{Study of problem (36)}

We first observe that, for $p \in S_{\delta}$ the semi-norm $\|\nabla p\|_{X^{\delta}}$ simplifies to

$$
\|\nabla p\|_{X^{\delta}}^{2}=\|\nabla p\|_{L^{2}\left(\Omega_{\alpha \delta}^{ \pm}\right)}^{2}+\delta\left\|\nabla_{\Gamma}\langle p\rangle_{\alpha}\right\|_{L^{2}(\Gamma)}^{2}+\frac{1}{\delta}\left\|\mathcal{G}_{\omega}\left(\operatorname{curl}_{\Gamma}[p]_{\alpha}\right)\right\|_{H\left(\operatorname{curl}_{\Gamma}, \Gamma\right)}^{2} .
$$

Thanks to Proposition 7, we deduce that $p \mapsto\|\nabla p\|_{X^{\delta}}$ defines a norm on $S^{\delta}$ equivalent to (31). We next observe that

$$
\begin{aligned}
b_{\delta}(\nabla p, \nabla q)= & \omega^{2} \int_{\Omega_{\alpha \delta}^{ \pm}} \nabla p \cdot \nabla q+\omega^{2} \delta \int_{\Gamma} \mathbf{D}_{m}\left\langle\nabla_{\Gamma} p\right\rangle_{\alpha} \cdot\left\langle\nabla_{\Gamma} q\right\rangle_{\alpha} d s \\
& -\frac{\omega^{2}}{\delta}\left\langle\mathcal{G}_{\omega}\left([\overrightarrow{\operatorname{curl}} \Gamma p]_{\alpha}\right),\left[\overrightarrow{\operatorname{curl}}_{\Gamma} q\right]_{\alpha}\right\rangle_{\Gamma}
\end{aligned}
$$

from which we deduce that solving (36) is equivalent to solving a non standard (and non coercive) transmission problem for the Laplace equation in $\Omega_{\delta}^{ \pm}$. Using (14), one gets

$$
\begin{aligned}
b_{\delta}(\nabla p, \nabla q)= & \omega^{2} \int_{\Omega_{\alpha \delta}^{ \pm}} \nabla p \cdot \nabla q+\omega^{2} \delta \int_{\Gamma} \mathbf{D}_{m}\left\langle\nabla_{\Gamma} p\right\rangle_{\alpha} \cdot\left\langle\nabla_{\Gamma} q\right\rangle_{\alpha} d s \\
& +\frac{A_{e} \omega^{2}}{\delta} \int_{\Gamma} \operatorname{curl}_{\Gamma} \mathcal{G}_{\omega}\left(\left[\operatorname{curl}_{\Gamma} p\right]_{\alpha}\right) \overrightarrow{\operatorname{curl}_{\Gamma} \mathcal{G}_{\omega}\left(\left[\operatorname{curl}_{\Gamma} q\right]_{\alpha}\right)} d s \\
& -\frac{\omega^{4}}{\delta} \int_{\Gamma} \mathbf{D}_{e} \mathcal{G}_{\omega}\left(\left[\overrightarrow{\left.\left.\operatorname{curl}_{\Gamma} p\right]_{\alpha}\right)}\right) \overrightarrow{\mathcal{G}_{\omega}\left(\left[\operatorname{curl}_{\Gamma} q\right]_{\alpha}\right)} d s\right.
\end{aligned}
$$

Thanks to Babuska's theory (see theorem 17), the well-posedness of problem (36) is a consequence of the following inf-sup conditions 
Proposition 10 (Inf-Sup condition for $b_{\delta}$ ). Assume that Hypothesis 6 holds. There exist $\delta_{0}>0$ and $C>0$ such that for all positive $\delta<\delta_{0}$,

$\|\nabla p\|_{X^{\delta}} \leq C \sup _{q \in S^{\delta} \backslash\{0\}} \frac{b_{\delta}(\nabla p, \nabla q)}{\|\nabla q\|_{X^{\delta}}}, \quad\|\nabla p\|_{X^{\delta}} \leq C \sup _{q \in S^{\delta} \backslash\{0\}} \frac{b_{\delta}(\nabla q, \nabla p)}{\|\nabla q\|_{X^{\delta}}} \quad \forall p \in S^{\delta}$.

Proof. By symmetry it suffices to prove the first inequality in (40), for which we employ a contradiction argument. If this inequality were not true, there would exists a sequence $p_{\delta} \in S_{\delta}$ such that

$$
\left\|\nabla p_{\delta}\right\|_{X^{\delta}}=1 \quad \text { and } \quad \lim _{\delta \rightarrow 0} \sup _{q \in S^{\delta} \backslash\{0\}} \frac{b_{\delta}\left(\nabla p_{\delta}, \nabla q\right)}{\|\nabla q\|_{X^{\delta}}}=0 .
$$

In order to work with fixed domains, we introduce the bijective mappings (simply translation-dilatations in the $x_{3}$ variable)

$$
F_{ \pm}^{\delta}:\left(x_{1}, x_{2}, x_{3}\right) \in \Omega^{ \pm}\left(:=\Omega_{0}^{ \pm}\right) \quad \mapsto \quad\left(x_{1}, x_{2},\left(1-\frac{2 \alpha \delta}{L_{3}}\right) x_{3} \pm \alpha \delta\right) \in \Omega_{\alpha \delta}^{ \pm}
$$

with constant Jacobians

$$
D F_{ \pm}^{\delta}=\operatorname{diag}\left\{1,1,\left(1-\frac{2 \alpha \delta}{L_{3}}\right)\right\} \quad:=\left[M_{ \pm}^{\delta}\right]^{-1}, \quad \text { with } \operatorname{det}\left[M_{ \pm}^{\delta}\right]^{-1}=1-\frac{2 \alpha \delta}{L_{3}} .
$$

For a function $q$ defined on $\Omega_{\alpha \delta}^{ \pm}$we define $\hat{q}$ on $\Omega^{ \pm}$by

$$
\hat{q}(x)=q\left(F_{ \pm}^{\delta}(x)\right) \quad x \in \Omega^{ \pm} .
$$

It is obvious that if $q \in S^{\delta}$ then $\hat{q} \in S^{0}$ (namely $S^{\delta}$ for $\delta=0$ ).

In the space $S^{0}$, we shall work with a $\delta$-dependent norm that is inspired from (37) by simply taking $\alpha=0$, namely $\left([\cdot]\right.$ and $\langle\cdot\rangle$ respectively refers to $[\cdot]_{0}$ and $\left.\langle\cdot\rangle_{0}\right)$

$$
\|\nabla \hat{q}\|_{\hat{X}^{\delta}}^{2}:=\|\nabla \hat{q}\|_{L^{2}\left(\Omega^{ \pm}\right)}^{2}+\delta\left\|\nabla_{\Gamma}\langle\hat{q}\rangle\right\|_{L^{2}(\Gamma)}^{2}+\frac{1}{\delta}\left\|\left[\mathcal{G}_{\omega}\left(\operatorname{curl}_{\Gamma}[\hat{q}]\right)\right]\right\|_{H\left(\operatorname{curl}_{\Gamma}, \Gamma\right)}^{2} .
$$

Since $F_{ \pm}^{\delta}$ "converges to identity" when $\delta \longrightarrow 0$, it is not difficult to guess and prove (the details are left to the reader) the following inequalities, as soon as $q$ and $\hat{q}$ are related by (44):

$$
(1-O(\delta))\|\nabla q\|_{X^{\delta}}^{2} \leq\|\nabla \hat{q}\|_{\hat{X}^{\delta}}^{2} \leq(1+O(\delta))\|\nabla q\|_{X^{\delta}}^{2},
$$

where $O(\delta)$ is independent of $q$.

Let us also define the sesquilinear $\hat{s}_{\delta}$ on $S^{0} \times S^{0}$ by

$$
\hat{s}_{\delta}(\hat{p}, \hat{q}):=b_{\delta}(\nabla p, \nabla q)
$$

Using the change of variable $x \longrightarrow F_{ \pm}^{\delta}(x)$ in (38), one gets, thanks to (43)

$$
\begin{aligned}
\hat{s}_{\delta}(\hat{p}, \hat{q}) & =\omega^{2}\left(1-\frac{2 \alpha \delta}{L_{3}}\right)^{-1} \int_{\Omega^{ \pm}} M_{ \pm}^{\delta} \nabla \hat{p} \cdot M_{ \pm}^{\delta} \overline{\nabla \hat{q}} d x \\
& +\omega^{2} \delta \int_{\Gamma} \mathbf{D}_{m}\left\langle\nabla_{\Gamma} \hat{p}\right\rangle \cdot \overline{\left\langle\nabla_{\Gamma} \hat{q}\right\rangle} d s-\frac{\omega^{2}}{\delta}\left\langle\mathcal{G}_{\omega}\left[\operatorname{curl}_{\Gamma} \hat{p}\right],\left[\operatorname{curl}_{\Gamma} \hat{q}\right]\right\rangle_{\Gamma} .
\end{aligned}
$$


or equivalently (see also (14))

$$
\begin{aligned}
\hat{s}_{\delta}(\hat{p}, \hat{q})= & \omega^{2}\left(1-\frac{2 \alpha \delta}{L_{3}}\right)^{-1} \int_{\Omega^{ \pm}} M_{ \pm}^{\delta} \nabla \hat{p} \cdot M_{ \pm}^{\delta} \overrightarrow{\nabla \hat{q}}+\omega^{2} \delta \int_{\Gamma} \mathbf{D}_{m}\left\langle\nabla_{\Gamma} p\right\rangle_{\alpha} \cdot\left\langle\nabla_{\Gamma} q\right\rangle_{\alpha} d s \\
& +\frac{A_{e} \omega^{2}}{\delta} \int_{\Gamma} \operatorname{curl}_{\Gamma} \mathcal{G}_{\omega}\left(\left[\operatorname{curl}_{\Gamma} p\right]_{\alpha}\right) \overrightarrow{\operatorname{curl}_{\Gamma} \mathcal{G}_{\omega}\left(\left[\operatorname{curl}_{\Gamma} q\right]_{\alpha}\right)} d s \\
& -\frac{\omega^{4}}{\delta} \int_{\Gamma} \mathbf{D}_{e} \mathcal{G}_{\omega}\left(\left[\operatorname{curl}_{\Gamma} p\right]_{\alpha}\right) \cdot \overrightarrow{\mathcal{G}_{\omega}\left(\left[\operatorname{curl}_{\Gamma} q\right]_{\alpha}\right)} d s
\end{aligned}
$$

Thanks to (46), (41) is equivalent to (with obvious notation)

$$
\text { (i) } \lim _{\delta \rightarrow 0}\left\|\nabla \hat{p}_{\delta}\right\|_{\hat{X}^{\delta}}=1 \quad \text { and } \quad(i i) \quad \lim _{\delta \rightarrow 0} \sup _{\hat{q} \in S_{0} \backslash\{0\}} \frac{\hat{s}_{\delta}\left(\hat{p}_{\delta}, \hat{q}\right)}{\|\nabla \hat{q}\|_{\hat{X}^{\delta}}}=0 .
$$

We split the rest of the proof into two steps.

- Step 1: we prove that $v^{\delta}:=\delta^{-\frac{1}{2}} \mathcal{G}_{\omega}\left(\operatorname{curl}_{\Gamma}\left[\hat{p}_{\delta}\right]\right)$ goes to 0 in $L^{2}(\Gamma)$ as $\delta \rightarrow 0$.

This is the more delicate step. First, from (50)(i), we have

$$
\operatorname{curl}_{\Gamma} v^{\delta} \text { is bounded in } L^{2}(\Gamma)^{2} \text {. }
$$

Moreover, from property (15) and $\operatorname{div}_{\Gamma} \operatorname{curl}_{\Gamma}\left[\hat{p}_{\delta}\right]=0$, we deduce that

$$
\operatorname{div}_{\Gamma}\left(\mathbf{D}_{e} v^{\delta}\right)=0 \text { on } \Gamma \text {. }
$$

Finally, since $\left\langle\operatorname{curl}_{\Gamma}\left[\hat{p}_{\delta}\right], \mathbf{C}\right\rangle_{\Gamma}=0, \forall \mathbf{C} \in \mathbb{P}_{0}^{2}$ (this simply follows from the periodicity $\left(x_{1}, x_{2}\right)$ of functions in $\left.S^{0}\right)$, we deduce from property (16) that

$$
\left\langle\mathbf{D}_{e} v^{\delta}, \mathbf{C}\right\rangle=0, \quad \forall \mathbf{C} \in \mathbb{P}_{0}^{2} .
$$

As a consequence, $v^{\delta} \in H_{\#}^{1}(\Gamma)$ and (we omit the proof which is based on (19) )

$$
\left\|v^{\delta}\right\|_{H^{1}(\Gamma)} \leq C\left\|\operatorname{curl}_{\Gamma} v^{\delta}\right\|_{L^{2}(\Gamma)} \leq C
$$

Therefore, up to a change in the subsequence $\left(\hat{p}_{\delta}\right)$, we can assume that

$$
v^{\delta} \rightarrow v, \quad \text { weakly in } H_{\#}^{1}(\Gamma)^{2}, \quad \text { strongly in } L^{2}(\Gamma)^{2},
$$

where $v$ satisfies (because of (52) and (53))

$$
\operatorname{div}_{\Gamma}\left(\mathbf{D}_{e} v\right)=0, \quad\left\langle\mathbf{D}_{e} v, \mathbf{C}\right\rangle=0, \quad \forall \mathbf{C} \in \mathbb{P}_{0}^{2} .
$$

We next prove that $v=0$. From (45), we see that, for any fixed $\hat{q}$ in $S^{0}, \delta^{-\frac{1}{2}}\|\nabla \hat{q}\|_{\hat{X}^{\delta}}$ is bounded. Therefore, we infer from (50)(ii) that

$$
\forall \hat{q} \in S^{0}, \quad \lim _{\delta \rightarrow 0} \delta^{\frac{1}{2}} \hat{s}_{\delta}\left(\hat{p}_{\delta}, \hat{q}\right)=0
$$

Using (48) and the definition of $v^{\delta}$ we can write

$$
\begin{aligned}
\delta^{\frac{1}{2}} \hat{s}_{\delta}\left(\hat{p}^{\delta}, \hat{q}\right) & =\omega^{2} \delta^{\frac{1}{2}}\left(1-\frac{2 \alpha \delta}{L_{3}}\right)^{-1} \int_{\Omega^{ \pm}} M_{ \pm}^{\delta} \nabla \hat{p}^{\delta} \cdot M_{ \pm}^{\delta} \bar{\nabla} \hat{q} \\
& +\omega^{2} \delta^{\frac{3}{2}} \int_{\Gamma} \mathbf{D}_{m}\left\langle\nabla_{\Gamma} \hat{p}^{\delta}\right\rangle \cdot \overline{\left\langle\nabla_{\Gamma} \hat{q}\right\rangle} d s-\omega^{2}\left\langle v^{\delta},\left[\operatorname{curl} l_{\Gamma} \hat{q}\right]\right\rangle_{\Gamma} .
\end{aligned}
$$


Passing to the limit when $\delta \rightarrow 0$ in (57), we get thanks to (56)

$$
\left\langle v^{\delta}, \operatorname{curl}_{\Gamma}[\hat{q}]\right\rangle_{\Gamma}=0, \quad \forall \hat{q} \in S^{0} .
$$

This obviously leads to (simply note that choosing $\hat{q}(x)=\chi\left(x_{3}\right) \varphi\left(x_{1}, x_{2}\right)$ if $x \in \Omega^{+}$ and $\hat{q}(x):=0$ if $x \in \Omega^{-}$, with $\chi \in C^{\infty}\left(0, L_{3}\right), \chi(0)=1, \chi\left(L_{3}\right)=0$ a given cut-off function and $\varphi$ is any function in $C_{0}^{\infty}(\Gamma)$, then $\hat{q} \in S^{0}$ and $[\hat{q}]=\varphi$ )

$$
\operatorname{curl}_{\Gamma} v=0
$$

which, combined with (55) yields $v=0$.

- Step 2: we get a contradiction. We now observe by comparing (45) and (39) that

$$
\begin{aligned}
\lim _{\delta \rightarrow 0}\left\|\hat{p}^{\delta}\right\|_{\hat{X}^{\delta}}^{2} & \leq C \lim _{\delta \rightarrow 0}\left(\hat{s}_{\delta}\left(\hat{p}_{\delta}, \hat{p}_{\delta}\right)+\frac{1}{\delta} \int_{\Gamma} \mathbf{D}_{e} \mathcal{G}_{\omega}\left(\operatorname{curl}_{\Gamma}\left[\hat{p}_{\delta}\right]\right) \cdot \overrightarrow{\left.\mathcal{G}_{\omega}\left(\overrightarrow{\operatorname{curl}} \Gamma \hat{p}_{\delta}\right]\right)}\right) \\
& \leq C \lim _{\delta \rightarrow 0}\left(\hat{s}_{\delta}\left(\hat{p}_{\delta}, \hat{p}_{\delta}\right)+\left\|v^{\delta}\right\|_{L^{2}(\Gamma)}^{2}\right)=0,
\end{aligned}
$$

since $\hat{s}_{\delta}\left(\hat{p}_{\delta}, \hat{p}_{\delta}\right) \longrightarrow 0$ by $(50)$ and $\left\|v^{\delta}\right\|_{L^{2}(\Gamma)}^{2} \longrightarrow 0$ by step 1 . This contradicts hypothesis (50)(i).

\subsection{Proof of the Helmholtz decomposition (Proposition 9)}

With the help of Proposition 10, the proof of the Helmholtz decomposition is a rather classical exercise that we reproduce here for the reader convenience. Our proof follows the lines of Ref. [29], Lemma 10.3. We already observed that $\nabla S^{\delta} \subset X^{\delta}$. The subspace $X_{0}^{\delta}$ is closed since $b_{\delta}$ is trivially continuous on $X^{\delta} \times X^{\delta}$. Let $\boldsymbol{\psi} \in X^{\delta}$. We define $p \in S^{\delta}$ solution to

$$
b_{\delta}(\nabla p, \nabla q)=b_{\delta}(\boldsymbol{\psi}, \nabla q), \quad \forall q \in S^{\delta}
$$

Since $q \mapsto b_{\delta}(u, \nabla q)$ is continuous on $S^{\delta}$, thanks to Proposition 10 , the solution $p$ exists for sufficiently small $\delta$. We define $\boldsymbol{\psi}_{0}=\boldsymbol{\psi}-\nabla p$. Clearly $\boldsymbol{\psi}_{0} \in X_{0}^{\delta}$. We now prove that $\left(\boldsymbol{\psi}_{0}, p\right)$ is unique. For that it would be sufficient to prove that if $\boldsymbol{\psi} \in$ $X_{0}^{\delta} \cap \nabla S^{\delta}$ then $\boldsymbol{\psi}=0$. Assume that $u \in X_{0}^{\delta}$ and $u=\nabla p$ for some $p \in S^{\delta}$. Then,

$$
b_{\delta}(\nabla p, \nabla q)=b_{\delta}(u, \nabla q)=0, \quad \forall q \in S^{\delta},
$$

since $u \in X_{0}^{\delta}$. Proposition 10 implies that $p=0$ and therefore $u=0$. The uniform continuity of the projection operators comes from the observation that (cf. also (29))

$$
\forall q \in S^{\delta} \text { and } \forall u \in X^{\delta}, \quad\left|b_{\delta}(u, \nabla q)\right| \leq C\|u\|_{X^{\delta}}\|\nabla q\|_{X^{\delta}}
$$

for some constant $C$ independent of $\delta$. Combined with the stability result of Proposition 10 , this implies that there exists $\delta_{0}>0$ and a constant $C>0$ such that

$$
\|\nabla p\|_{X^{\delta}} \leq C\|\boldsymbol{\psi}\|_{X^{\delta}}
$$

for all $\boldsymbol{\psi} \in X^{\delta}, \boldsymbol{\psi}=\boldsymbol{\psi}_{0}+\nabla p$ and $p \in S^{\delta}$. 


\subsection{Properties of $X_{0}^{\delta}$}

We shall prove in this section the central point behind the Helmholtz decomposion, namely the collective compact embedding of the spaces $X_{0}^{\delta}$ in $L^{2}\left(\Omega_{\alpha \delta}^{ \pm}\right)^{3}$. The latter is a direct consequence of the following proposition and classical Sobolev compact embedding theorems.

Proposition 11. Assume Hypothesis 6 and let $0<\delta<L_{3} / 4 \alpha$. Then $X_{0}^{\delta} \subset H^{1 / 2}\left(\Omega_{\alpha \delta}^{ \pm}\right)^{3}$. Moreover, there exists a constant $C$ independent of $\delta$ such that, for any $\psi \in X_{0}^{\delta}$,

$$
\|\boldsymbol{\psi}\|_{H^{1 / 2}\left(\Omega_{\alpha \delta}^{ \pm}\right)}^{2} \leq C\|\boldsymbol{\psi}\|_{X^{\delta}} .
$$

Remark 12. The restriction $\delta<L_{3} / 4 \alpha$ is essentially indicative. Moreover, the reader would easily observe that functions in $X_{0}^{\delta}$ have $H^{1}$ regularity except in the neighborhood of $\Sigma_{3}^{ \pm}$(it suffices to "play" with the cut-off function $\chi$ in the proof of Proposition 11). The global $H^{\frac{1}{2}}$ restriction is due to imposing $L^{2}\left(\Sigma_{3}^{ \pm}\right)$regularity on tangential traces on $\Sigma_{3}^{ \pm}$(due to impedance conditions on $\Sigma_{3}^{ \pm}$).

The proof of Proposition 11 will use the following two technical lemmas 13 and 14 and an intermediate result, the main one in fact, that concern functions in $X_{0}^{\delta}$ that "vanish" on $\Sigma_{3}^{ \pm}$(cf (35) and lemma 15).

Lemma 13. Let $\psi \in H_{\#}\left(\operatorname{div}, \Omega_{\alpha \delta}^{ \pm}\right) \cap H_{\#}\left(\operatorname{curl}, \Omega_{\alpha \delta}^{ \pm}\right)$satisfying $\psi \times e_{3}=0$ on $\Sigma_{3}^{ \pm}$ and such that

$$
\left\langle\boldsymbol{\psi}_{T}\right\rangle_{\alpha} \in H^{1 / 2}(\Gamma) \text { and }\left\langle\boldsymbol{\psi} \cdot e_{3}\right\rangle_{\alpha} \in H^{1 / 2}(\Gamma) .
$$

Then $\psi \in H^{1}\left(\Omega_{\alpha \delta}^{ \pm}\right)^{3}$.

Proof. The general idea is to use the fact that vector fields in $H$ (div) $\cap H_{\#}$ (curl) are in fact the $H^{1}$ regularity as soon as one of their traces, either the normal trace (see Theorem 5.4.3 in Ref. [30]) or the tangential trace (see Ref. [5], Remark 2.14) are in $H^{1 / 2}$. Our lemma essentially expresses that we get a similar result for "transmission problems" provided additional regularity on only the mean values of all the traces from both sides of the interface.

Let $\psi$ satisfying the assumptions of the lemma and $\psi^{ \pm}$be the restriction of $\psi$ to $\Omega_{\alpha \delta}^{ \pm}$.

We construct $\phi$ on $\Omega_{\alpha \delta}^{-}$from $\psi^{+}$by symmetry or anti-symmetry depending of the component:

$$
\begin{aligned}
& \phi_{i}\left(x_{1}, x_{2}, x_{3}\right)=\boldsymbol{\psi}_{i}^{+}\left(x_{1}, x_{2},-x_{3}\right), \quad i=1,2, \\
& \phi_{3}\left(x_{1}, x_{2}, x_{3}\right)=-\boldsymbol{\psi}_{3}^{+}\left(x_{1}, x_{2},-x_{3}\right)
\end{aligned}
$$

for all $x \in \Omega_{\alpha \delta}^{-}$. Clearly $\phi \in H_{\#}\left(\operatorname{div}, \Omega_{\alpha \delta}^{-}\right) \cap H_{\#}\left(\operatorname{curl}, \Omega_{\alpha \delta}^{-}\right)$. We define $\varphi$ on $\Omega_{\alpha \delta}^{-}$ by

$$
\varphi:=\psi^{-}-\phi
$$

By construction, $\varphi \in H_{\#}\left(\operatorname{div}, \Omega_{\alpha \delta}^{-}\right) \cap H_{\#}\left(\operatorname{curl}, \Omega_{\alpha \delta}^{-}\right)$. In addition, $\varphi \times e_{3}=0$ on $\Sigma_{3}^{-}$and

$$
\left(\boldsymbol{\varphi} \cdot e_{3}\right)_{\alpha}^{-}=2\left\langle\boldsymbol{\psi} \cdot e_{3}\right\rangle_{\alpha} \in H^{1 / 2}(\Gamma) .
$$

Consequently $\varphi \in H^{1}\left(\Omega_{\alpha \delta}^{-}\right)^{3}$ (Theorem 5.4.3 in Ref. [30]). We deduce that $\left[\boldsymbol{\psi}_{T}\right]_{\alpha}=$ $\left(\boldsymbol{\varphi}_{T}\right)_{\alpha}^{-}$belongs to $H^{1 / 2}(\Gamma)^{3}$. Since $\left\langle\psi_{T}\right\rangle_{\alpha}$ also belongs to $H^{1 / 2}(\Gamma)^{3}$, we conclude that $\left(\boldsymbol{\psi}_{T}\right)_{\alpha}^{ \pm}$are in $H^{1 / 2}(\Gamma)^{3}$. Hence $\boldsymbol{\psi} \in H^{1}\left(\Omega_{\alpha \delta}^{ \pm}\right)^{3}$ (see for instance Remark 2.14 in Ref. [5]). 
Lemma 14. Let $\mathbf{D}$ be a $2 \times 2$ positive definite and symmetric matrix. Then, there exists a positive constant $C$ such that $(\mathcal{R} e(u)$ denotes the real part of $u$ )

$$
\forall u \in H_{\#}^{1}(\Gamma), \quad-\mathcal{R} e\left(\int_{\Gamma} \operatorname{div}_{\Gamma}(\mathbf{D} u) \overline{\operatorname{div}_{\Gamma} u} d s\right) \leq C\left\|\operatorname{curl}_{\Gamma} u\right\|_{L^{2}(\Gamma)}^{2} .
$$

Proof. Using Fourier series, proving (63) amounts to proving that:

$$
\forall \mathbf{k} \in \frac{2 \pi}{L_{1}} \mathbb{Z} \times \frac{2 \pi}{L_{2}} \mathbb{Z}, \quad \forall v \in \mathbb{C}^{2}, \quad-\mathcal{R} e((\mathbf{k} \cdot \mathbf{D} v)(\mathbf{k} \cdot \bar{v})) \leq C|\mathbf{k} \times v|^{2} .
$$

We can choose to work in the eigenbasis of $\mathbf{D}$, which amounts to consider that $\mathbf{D}$ is diagonal: $\mathbf{D}=\operatorname{diag}\left\{d_{1}, d_{2}\right\}$ with $\left(d_{1}, d_{2}\right) \in \mathbb{R}_{*}^{+}$. In this case

$-\mathcal{R} e((\mathbf{k} \cdot \mathbf{D} v)(\mathbf{k} \cdot \bar{v}))=-d_{1}\left|\mathbf{k}_{1}\right|^{2}\left|v_{1}\right|^{2}-d_{2}\left|\mathbf{k}_{2}\right|^{2}\left|v_{2}\right|^{2}-\left(d_{1}+d_{2}\right) \mathbf{k}_{1} \mathbf{k}_{2} \mathcal{R} e\left(v_{1} \overline{v_{2}}\right)$

In particular, $d_{1}$ and $d_{2}$ being positive,

$$
-\mathcal{R} e((\mathbf{k} \cdot \mathbf{D} v)(\mathbf{k} \cdot \bar{v})) \leq-\left(d_{1}+d_{2}\right) \mathcal{R} e\left[\left(\mathbf{k}_{2} v_{1}\right)\left(\mathbf{k}_{1} \overline{v_{2}}\right)\right]
$$

Writing $\mathbf{k}_{2} v_{1}=\left(\mathbf{k}_{2} v_{1}-\mathbf{k}_{1} v_{2}\right)+\mathbf{k}_{1} v_{2}$, we get

$$
-\mathcal{R} e((\mathbf{k} \cdot \mathbf{D} v)(\mathbf{k} \cdot \bar{v})) \leq-\left(d_{1}+d_{2}\right)\left(\mathcal{R} e\left[(\mathbf{k} \times v)\left(\mathbf{k}_{1} \overline{v_{2}}\right)\right]+\left|\mathbf{k}_{1}\right|^{2}\left|v_{2}\right|^{2}\right)
$$

which yields

$$
-\mathcal{R} e((\mathbf{k} \cdot \mathbf{D} v)(\mathbf{k} \cdot \bar{v})) \leq-\left(d_{1}+d_{2}\right)\left|\mathbf{k}_{1}\right|^{2}\left|v_{2}\right|^{2}+\left(d_{1}+d_{2}\right)|\mathbf{k} \times v|\left|\mathbf{k}_{1} v_{2}\right|
$$

By Young's inequality $|\mathbf{k} \times v|\left|\mathbf{k}_{1} v_{2}\right| \leq \eta|\mathbf{k} \times v|^{2}+(1 / 4 \eta)\left|\mathbf{k}_{1} v_{2}\right|^{2}$ for any $\eta>0$. Thus

$-\mathcal{R} e((\mathbf{k} \cdot \mathbf{D} v)(\mathbf{k} \cdot \bar{v})) \leq\left(\frac{1}{4 \eta}-1\right)\left(d_{1}+d_{2}\right)\left|\mathbf{k}_{1}\right|^{2}\left|v_{2}\right|^{2}+\eta\left(d_{1}+d_{2}\right)|\mathbf{k} \times v|\left|\mathbf{k}_{1} v_{2}\right|$ so that choosing $\eta=1 / 4$ leads to (64) with $C=\frac{d_{1}+d_{2}}{4}$. This concludes the proof.

Let us introduce the space $\tilde{X}^{\delta}$ defined by (note that this space differs from $X^{\delta}$ by the divergence condition and the boundary condition on $\Sigma_{3}^{ \pm}$):

$$
\begin{aligned}
\tilde{X}^{\delta}= & \left\{\boldsymbol{\psi} \in X^{\delta}, \operatorname{div} \boldsymbol{\psi} \in L^{2}\left(\Omega_{\alpha \delta}^{ \pm}\right),\left.\boldsymbol{\psi} \cdot e_{i}\right|_{\Sigma_{i, \delta}^{+}}=\left.\boldsymbol{\psi} \cdot e_{i}\right|_{\Sigma_{i, \delta}^{-}}, i=1,2, \boldsymbol{\psi} \times\left. e_{3}\right|_{\Sigma_{3}^{ \pm}}=0,\right. \\
& \left.\left\langle\boldsymbol{\psi} \cdot e_{3}\right\rangle_{\alpha}=\frac{1}{\delta} \operatorname{curl}_{\Gamma} \mathcal{G}_{\omega}\left(\left[e_{3} \times \boldsymbol{\psi}\right]_{\alpha}\right) \text { and }-\left[\boldsymbol{\psi} \cdot e_{3}\right]_{\alpha}=\delta \operatorname{div}_{\Gamma}\left[\mathbf{D}_{e}\left\langle\boldsymbol{\psi}_{T}\right\rangle_{\alpha}\right] \text { on } \Gamma\right\} .
\end{aligned}
$$

Lemma 15. The space $\tilde{X}^{\delta}$ is embedded into $H^{1}\left(\Omega_{\alpha \delta}^{ \pm}\right)^{3}$ for $\delta<L_{3} / 4 \alpha$. Moreover, there exists a constant $C>0$ independent of $\delta$ such that

$$
\forall \boldsymbol{\psi} \in \tilde{X}^{\delta}, \quad\|\boldsymbol{\psi}\|_{H^{1}\left(\Omega_{\alpha \delta}^{ \pm}\right)^{3}}^{2} \leq C\left(\|\boldsymbol{\psi}\|_{\tilde{X}^{\delta}}^{2}+\frac{1}{\delta}\left\|\mathcal{G}_{\omega}\left(\left[e_{3} \times \boldsymbol{\psi}\right]_{\alpha}\right)\right\|_{L^{2}(\Gamma)}^{2}\right)
$$

where $\|\boldsymbol{\psi}\|_{\tilde{X}^{\delta}}^{2}:=\|\boldsymbol{\psi}\|_{L^{2}\left(\Omega_{\alpha \delta}^{ \pm}\right)^{3}}^{2}+\|\operatorname{div} \boldsymbol{\psi}\|_{L^{2}\left(\Omega_{\alpha \delta}^{ \pm}\right)}^{2}+\|\operatorname{curl} \boldsymbol{\psi}\|_{L^{2}\left(\Omega_{\alpha \delta}^{ \pm}\right)^{3}}^{2}+\delta\left\|\operatorname{curl} l_{\Gamma}\left\langle\boldsymbol{\psi}_{T}\right\rangle_{\alpha}\right\|_{L^{2}(\Gamma)}^{2}$. 
Proof. Let $\psi \in \tilde{X}^{\delta}$. The transmission conditions

$$
\left\langle\boldsymbol{\psi} \cdot e_{3}\right\rangle_{\alpha}=\frac{1}{\delta} \operatorname{curl}_{\Gamma} \mathcal{G}_{\omega}\left(\left[e_{3} \times \boldsymbol{\psi}_{T}\right]_{\alpha}\right) \text { and }-\left[\boldsymbol{\psi} \cdot e_{3}\right]_{\alpha}=\delta \operatorname{div}_{\Gamma}\left(\mathbf{D}_{m}\left\langle\boldsymbol{\psi}_{T}\right\rangle_{\alpha}\right)
$$

infer a higher regularity than $H^{-1 / 2}$ for $\langle\boldsymbol{\psi}\rangle_{\Gamma}$. Indeed, from the definition of $\mathcal{G}_{\omega}$ we know that

$$
\operatorname{curl}_{\Gamma} \operatorname{curl}_{\Gamma} \mathcal{G}_{\omega}\left(\left[e_{3} \times \boldsymbol{\psi}_{T}\right]_{\alpha}\right) \in H_{\#}^{-1 / 2}(\Gamma)^{3} .
$$

Since in addition, $\left\langle\boldsymbol{\psi} \cdot e_{3}\right\rangle_{\alpha}$ is also in $H_{\#}^{-1 / 2}(\Gamma)$ and $\operatorname{curl}_{\Gamma}\left\langle\boldsymbol{\psi} \cdot e_{3}\right\rangle_{\alpha}=\frac{1}{\delta} \overrightarrow{\operatorname{curl}}_{\Gamma} \operatorname{curl}_{\Gamma} \mathcal{G}_{\omega}\left(\left[e_{3} \times\right.\right.$ $\left.\left.\boldsymbol{\psi}_{T}\right]_{\alpha}\right)$, then

$$
\left\langle\boldsymbol{\psi} \cdot e_{3}\right\rangle_{\alpha} \in H_{\#}^{1 / 2}(\Gamma) .
$$

On the other hand, $\operatorname{div}_{\Gamma}\left(\mathbf{D}_{m}\left\langle\boldsymbol{\psi}_{T}\right\rangle_{\alpha}\right)=-(1 / \delta)\left[\boldsymbol{\psi} \cdot e_{3}\right]_{\alpha} \in H_{\#}^{-1 / 2}(\Gamma)^{3}$ while $\left\langle\boldsymbol{\psi}_{T}\right\rangle_{\alpha} \in$ $H_{\#}^{1 / 2}\left(\operatorname{curl}_{\Gamma}, \Gamma\right)$ by trace theorem. Therefore, using again (19), we deduce that

$$
\left\langle\boldsymbol{\psi}_{T}\right\rangle_{\alpha} \in H_{\#}^{1 / 2}(\Gamma) .
$$

We conclude by applying Lemma 13 that $\psi \in H^{1}\left(\Omega_{\alpha \delta}^{ \pm}\right)^{3}$ (for fixed $\delta$ ).

To evaluate the $L^{2}$ norm of $\nabla \boldsymbol{\psi}$, we use the following identities (adapted from the one in Ref. [30], page 211), which are easily obtained by repeated integration by parts

$$
\begin{aligned}
\|\nabla \boldsymbol{\psi}\|_{L^{2}\left(\Omega_{\alpha \delta}^{+}\right)}^{2} & =\int_{\Omega_{\alpha \delta}^{+}}\left(|\operatorname{curl} \boldsymbol{\psi}|^{2}+|\operatorname{div} \boldsymbol{\psi}|^{2}\right) d x+2 \mathcal{R} e\left\langle\operatorname{div}_{\Gamma}\left(\boldsymbol{\psi}_{T}\right)_{\alpha}^{+},\left(\boldsymbol{\psi} \cdot e_{3}\right)_{\alpha}^{+}\right\rangle_{\Gamma}, \\
\|\nabla \boldsymbol{\psi}\|_{L^{2}\left(\Omega_{\alpha \delta}^{-}\right)}^{2} & =\int_{\Omega_{\alpha \delta}^{-}}\left(|\operatorname{curl} \boldsymbol{\psi}|^{2}+|\operatorname{div} \boldsymbol{\psi}|^{2}\right) d x-2 \mathcal{R} e\left\langle\operatorname{div}_{\Gamma}\left(\boldsymbol{\psi}_{T}\right)_{\alpha}^{-},\left(\boldsymbol{\psi} \cdot e_{3}\right)_{\alpha}^{-}\right\rangle_{\Gamma},
\end{aligned}
$$

where $\mathcal{R} e$ indicates the real part. Summing the last two equalities proves that

$$
\begin{aligned}
& \|\nabla \boldsymbol{\psi}\|_{L^{2}\left(\Omega_{\alpha \delta}^{ \pm}\right)}^{2}=\int_{\Omega_{\alpha \delta}^{ \pm}}\left(|\operatorname{curl} \boldsymbol{\psi}|^{2}+|\operatorname{div} \boldsymbol{\psi}|^{2}\right) d x \\
& \quad+2 \mathcal{R} e\left(\left\langle\operatorname{div}_{\Gamma}\left[\boldsymbol{\psi}_{T}\right]_{\alpha},\left\langle\boldsymbol{\psi} \cdot e_{3}\right\rangle_{\alpha}\right\rangle_{\Gamma}+\left\langle\operatorname{div}_{\Gamma}\left\langle\boldsymbol{\psi}_{T}\right\rangle_{\alpha},\left[\boldsymbol{\psi} \cdot e_{3}\right]_{\alpha}\right\rangle_{\Gamma}\right) .
\end{aligned}
$$

Using first (67) and next lemma 14,

$$
\begin{aligned}
\mathcal{R} e\left\langle\operatorname{div}_{\Gamma}\left\langle\boldsymbol{\psi}_{T}\right\rangle_{\alpha},\left[\boldsymbol{\psi} \cdot e_{3}\right]_{\alpha}\right\rangle_{\Gamma} & =-\delta \mathcal{R} e\left(\left\langle\operatorname{div}_{\Gamma}\left\langle\boldsymbol{\psi}_{T}\right\rangle_{\alpha}, \operatorname{div}_{\Gamma} \mathbf{D}_{m}\left\langle\boldsymbol{\psi}_{T}\right\rangle_{\alpha}\right)\right. \\
& \leq C \delta\left\|\operatorname{curl}_{\Gamma}\left\langle\boldsymbol{\psi}_{T}\right\rangle_{\alpha}\right\|_{L^{2}(\Gamma)}^{2} .
\end{aligned}
$$

On the other hand, from the identity $\operatorname{div}_{\Gamma}\left[\boldsymbol{\psi}_{T}\right]_{\alpha}=\operatorname{curl}_{\Gamma}\left[e_{3} \times \boldsymbol{\psi}\right]_{\alpha}$ and (67) again

$$
\left\langle\operatorname{div}_{\Gamma}\left[\boldsymbol{\psi}_{T}\right]_{\alpha},\left\langle\boldsymbol{\psi} \cdot e_{3}\right\rangle_{\alpha}\right\rangle_{\Gamma}=\frac{1}{\delta}\left\langle\operatorname{curl}_{\Gamma}\left[e_{3} \times \boldsymbol{\psi}\right]_{\alpha}, \operatorname{curl}_{\Gamma} \mathcal{G}_{\omega}\left(\left[e_{3} \times \boldsymbol{\psi}\right]_{\alpha}\right)\right\rangle_{\Gamma}
$$

On the other hand, by the property (17) of $\mathcal{G}_{\omega}$,

$$
\begin{array}{r}
\mathcal{R} e\left\langle\operatorname{curl}_{\Gamma}\left[e_{3} \times \boldsymbol{\psi}\right]_{\alpha}, \operatorname{curl}_{\Gamma} \mathcal{G}_{\omega}\left(\left[e_{3} \times \boldsymbol{\psi}\right]_{\alpha}\right)\right\rangle_{\Gamma}=-\frac{1}{A_{e}} \int_{\Gamma}\left|\left[e_{3} \times \boldsymbol{\psi}\right]_{\alpha}\right|^{2} d s \\
+\mathcal{R} e\left(\frac{\omega^{2}}{A_{e}} \int_{\Gamma} \mathbf{D}_{e} \overline{\mathcal{G}_{\omega}\left(\left[e_{3} \times \boldsymbol{\psi}\right]_{\alpha}\right)} \cdot\left[e_{3} \times \boldsymbol{\psi}\right]_{\alpha} d s\right)
\end{array}
$$


By Young's inequality, for any $\eta>0$, there exists a constant $C>0$ such that

$$
\begin{aligned}
\left.\mid \frac{\omega^{2}}{A_{e}} \int_{\Gamma} \mathbf{D}_{e} \overline{\mathcal{G}_{\omega}\left(\left[e_{3} \times \boldsymbol{\psi}\right]_{\alpha}\right)} \cdot\left[e_{3} \times \boldsymbol{\psi}\right]_{\alpha}\right) d s \mid \leq \\
\quad \eta \int_{\Gamma}\left|\left[e_{3} \times \boldsymbol{\psi}\right]_{\alpha}\right|^{2} d s+\frac{C}{\eta} \int_{\Gamma}\left|\mathcal{G}_{\omega}\left[e_{3} \times \boldsymbol{\psi}\right]_{\alpha}\right|^{2} d s
\end{aligned}
$$

Subsituting this inequality in , with $\eta=-\frac{1}{A_{e}}$ gives, for some $C>0$ independent of $\delta$

$$
\mathcal{R} e\left\langle\operatorname{curl}_{\Gamma}\left[e_{3} \times \boldsymbol{\psi}\right]_{\alpha}, \operatorname{curl}_{\Gamma} \mathcal{G}_{\omega}\left(\left[e_{3} \times \boldsymbol{\psi}\right]_{\alpha}\right)\right\rangle_{\Gamma} \leq C\left\|\mathcal{G}_{\omega}\left(\left[e_{3} \times \boldsymbol{\psi}\right]_{\alpha}\right)\right\|_{L^{2}(\Gamma)}^{2}
$$

Therefore

$$
\mathcal{R} e\left\langle\operatorname{div}_{\Gamma}\left[\boldsymbol{\psi}_{T}\right]_{\alpha},\left\langle\boldsymbol{\psi} \cdot e_{3}\right\rangle_{\alpha}\right\rangle_{\Gamma} \leq \frac{C}{\delta}\left\|\mathcal{G}_{\omega}\left(\left[e_{3} \times \boldsymbol{\psi}\right]_{\alpha}\right)\right\|_{L^{2}(\Gamma)}^{2}
$$

Combining (68), (69) and (71) leads to (67), which achieves the proof.

Proof of Proposition 11. Let $\psi \in X^{\delta}$ and let $\chi$ be a $\mathcal{C}^{\infty}(\mathbb{R})$ cut-off function such that

$$
\chi\left(x_{3}\right)=1 \text { for }\left|x_{3}\right| \leq \frac{L_{3}}{4}, \quad \chi\left(x_{3}\right)=0 \text { for }\left|x_{3}\right| \geq \frac{L_{3}}{2} .
$$

We split $\boldsymbol{\psi}$ into the sum of $\boldsymbol{\psi}_{1}:=\chi \boldsymbol{\psi}$ and $\boldsymbol{\psi}_{2}=(1-\chi) \boldsymbol{\psi}$.

By construction $\boldsymbol{\psi}_{1} \in \tilde{X}_{0}^{\delta}$. Hence, applying the result of Lemma $15, \psi_{1} \in H^{1}\left(\Omega_{\alpha \delta}^{ \pm}\right)^{3}$ and satisfies

$$
\begin{array}{r}
\left\|\boldsymbol{\psi}_{1}\right\|_{H^{1}\left(\Omega_{\alpha \delta}^{ \pm}\right)}^{2} \leq C\left(\|\boldsymbol{\psi}\|_{H\left(\operatorname{curl}, \Omega_{\alpha \delta}^{ \pm}\right)}^{2}+\left\|\boldsymbol{\psi}_{T}\right\|_{L^{2}\left(\Sigma_{3}^{ \pm}\right)^{3}}^{2}+\delta\left\|\operatorname{curl}_{\Gamma}\left\langle\boldsymbol{\psi}_{T}\right\rangle_{\alpha}\right\|_{L^{2}(\Gamma)}^{2}\right. \\
\left.+\frac{1}{\delta}\left\|\mathcal{G}_{\omega}\left(\left[e_{3} \times \boldsymbol{\psi}\right]_{\alpha}\right)\right\|_{L^{2}(\Gamma)}^{2}\right)
\end{array}
$$

where we have used in particular $\operatorname{div} \boldsymbol{\psi}_{1}=\nabla \chi \cdot \boldsymbol{\psi}$ in $\Omega_{\alpha \delta}^{ \pm}$.

The function $\boldsymbol{\psi}_{2}$ belongs to $H_{\#}\left(\operatorname{curl}, \Omega_{\alpha \delta}^{ \pm}\right) \cap H_{\#}\left(\operatorname{div}, \Omega_{\alpha \delta}^{ \pm}\right)$, have a support that does not touch $\Sigma_{i, \delta}^{ \pm}$and have $L^{2}$ tangential traces on $\Sigma_{3}^{ \pm}$. Thus, a slight adaptation of Theorem 2 of Ref. [12] then implies that $\psi_{2} \in H^{1 / 2}\left(\Omega_{\alpha \delta}^{ \pm}\right)^{3}$ and also satisfies, since $\operatorname{div} \boldsymbol{\psi}_{2}=-\nabla \chi \cdot \boldsymbol{\psi}$

$$
\left\|\boldsymbol{\psi}_{2}\right\|_{H^{1 / 2}\left(\Omega_{\alpha \delta}^{ \pm}\right)}^{2} \leq C\left(\|\boldsymbol{\psi}\|_{H\left(\operatorname{curl}, \Omega_{\alpha \delta}^{ \pm}\right)}^{2}+\left\|\boldsymbol{\psi}_{T}\right\|_{L^{2}\left(\Sigma_{3}^{ \pm}\right)^{3}}^{2}\right)
$$

Thus $\boldsymbol{\psi}=\boldsymbol{\psi}_{1}+\boldsymbol{\psi}_{2} \in H^{1 / 2}\left(\Omega_{\alpha \delta}^{ \pm}\right)$and the estimate (61) follows from (72) and (73).

\section{Well-posedness and uniform stability results}

We are now in position to prove the first main result of this paper, namely the existence, uniqueness and uniform continuity (with respect to $\delta$ ) of the solutions to (21). 
Proposition 16. These exists $\delta_{0}>0$ and $C>0$ such that, for any $0<\delta<\delta_{0}$ and any $f \in L^{2}\left(\Omega_{\alpha \delta}^{+} \cup \Omega_{\alpha \delta}^{-}\right)^{3}$, the problem (21) admits a unique solution $E^{\delta} \in X^{\delta}$ satisfying the uniform estimate

$$
\left\|E^{\delta}\right\|_{X^{\delta}} \leq C\|f\|_{L^{2}\left(\Omega_{\alpha \delta}^{+} \cup \Omega_{\alpha \delta}^{-}\right)} .
$$

To prove these well-posedness and uniform stability results associated with (21), we shall use the well known Babuska's theory for variational problem.

Theorem 17. Let $V$ be an Hilbert space and $a(u, v)$ a continuous sesquilinear form on $V \times V$

$$
|a(u, v)| \leq M\|u\|_{V}\|v\|_{V}
$$

Assume that there exists $\beta>0$ such that

$$
\forall u \in V, \quad \sup _{v \in V} \frac{\mid a(u, v)}{\|v\|_{V}} \geq \beta\|u\|_{V}, \quad \forall v \in V, \quad \sup _{u \in V} \frac{\mid a(u, v)}{\|u\|_{V}} \geq \beta\|v\|_{V} .
$$

Then, for any $L \in V^{\prime}$ (the dual of space of $V$ ), the variational problem

$$
\text { Find } u \in V \text { such that } \forall v \in V, \quad a(u, v)=\langle L, v\rangle
$$

admits a unique solution which satisfies

$$
\|u\|_{V} \leq \frac{M}{\beta}\|L\|_{V^{\prime}}
$$

Applying theorem 17 with $V:=X_{0}^{\delta}, a:=a^{\delta}$ and

$$
<L, v>:=\int_{\Omega_{\alpha \delta}^{ \pm}} f \cdot \bar{v} d x,
$$

we see that, taking into account the uniform continuity of $a^{\delta}$ and the inequality

$$
\left.|<L, v>| \leq\|f\|_{L^{2}\left(\Omega_{\alpha \delta}^{+} \cup \Omega_{\alpha \delta}^{-}\right)}\|v\|_{X^{\delta}} \quad \text { (i.e. } \quad\|L\|_{\left(X^{\delta}\right)^{\prime}} \leq\|f\|_{L^{2}\left(\Omega_{\alpha \delta}^{+} \cup \Omega_{\alpha \delta}^{-}\right)}\right)
$$

the proposition (16) is a direct consequence of the following inf-sup conditions:

Proposition 18. Assume Hypothesis 6. There exist $\delta_{0}>0$ and $\beta>0$ such that for all positive $\delta<\delta_{0}$,

$$
\begin{aligned}
& \forall \boldsymbol{\psi} \in X^{\delta}, \quad \sup _{\boldsymbol{\varphi} \in X^{\delta}, \boldsymbol{\varphi \neq 0}} \frac{a_{\delta}(\boldsymbol{\psi}, \boldsymbol{\varphi})}{\|\boldsymbol{\varphi}\|_{X^{\delta}}} \geq \beta\|\boldsymbol{\psi}\|_{X^{\delta}} . \\
& \forall \boldsymbol{\varphi} \in X^{\delta}, \quad \sup _{\boldsymbol{\psi} \in X^{\delta}, \boldsymbol{\psi} \neq 0} \frac{a_{\delta}(\boldsymbol{\psi}, \boldsymbol{\varphi})}{\|\boldsymbol{\psi}\|_{X^{\delta}}} \geq \beta\|\boldsymbol{\varphi}\|_{X^{\delta}} .
\end{aligned}
$$

Proof. Considering the symmetry of $a^{\delta}$, it is clear that the proofs of (80) and (81) are identical. To prove (80), we shall use a technique similar to the one used in the proof of Proposition 10. Contradicting (80) is equivalent to the existence of $\delta \mapsto \boldsymbol{\psi}_{\delta} \in X^{\delta}$ such that

$$
\left\|\boldsymbol{\psi}_{\delta}\right\|_{X^{\delta}}=1 \quad \text { and } \quad \lim _{\delta \rightarrow 0} \sup _{\varphi \in X_{0}^{\delta}, \boldsymbol{\varphi} \neq 0} \frac{a_{\delta}\left(\boldsymbol{\psi}_{\delta}, \boldsymbol{\varphi}\right)}{\|\boldsymbol{\varphi}\|_{X^{\delta}}}=0
$$


According to the Helmholtz decomposition (Proposition 9), we can write

$$
\boldsymbol{\psi}_{\delta}=\boldsymbol{\psi}_{\delta}^{0}+\nabla p^{\delta}, \quad \boldsymbol{\psi}_{\delta}^{0} \in X_{0}^{\delta}, \quad p^{\delta} \in S^{\delta}
$$

where, by uniform continuity of the projections

$$
\left\|\boldsymbol{\psi}_{\delta}^{0}\right\|_{X^{\delta}}+\left\|\nabla p^{\delta}\right\| \leq C\left\|\boldsymbol{\psi}_{\delta}\right\|_{X^{\delta}}
$$

and where $p^{\delta}$ is uniquely defined by (see (36) and (32))

$$
\forall q \in S^{\delta}, \quad b^{\delta}\left(\nabla p^{\delta}, \nabla q\right)=a^{\delta}\left(\nabla p^{\delta}, \nabla q\right)=a^{\delta}\left(\boldsymbol{\psi}^{\delta}, \nabla q\right)
$$

which yields,

$$
\sup _{q \in S^{\delta}, q \neq 0} \frac{b^{\delta}\left(\nabla p^{\delta}, \nabla q\right)}{\|\nabla q\|_{X^{\delta}}} \leq \sup _{\boldsymbol{\varphi} \in X^{\delta}, \boldsymbol{\varphi \neq 0}} \frac{a_{\delta}\left(\boldsymbol{\psi}_{\delta}, \boldsymbol{\varphi}\right)}{\|\boldsymbol{\varphi}\|_{X^{\delta}}}
$$

From the inf-sup condition (40) and (82)

$$
\lim _{\delta \rightarrow 0}\left\|\nabla p^{\delta}\right\|_{X^{\delta}}=0 \quad \text { which implies } \quad \lim _{\delta \rightarrow 0}\left\|\boldsymbol{\psi}_{\delta}^{0}\right\|_{X^{\delta}}=1 \text {. }
$$

Decomposing any $\varphi \in X^{\delta}$ as $\varphi=\varphi^{0}+\nabla q, \varphi^{0} \in X_{0}^{\delta}, q \in S^{\delta}$, with $\left\|\varphi^{0}\right\|_{X^{\delta}} \leq$ $C\|\varphi\|_{X^{\delta}}$, we get

$\frac{a_{\delta}\left(\boldsymbol{\psi}_{\delta}, \boldsymbol{\varphi}\right)}{\|\boldsymbol{\varphi}\|_{X^{\delta}}}=\frac{a_{\delta}\left(\boldsymbol{\psi}_{\delta}^{0}, \boldsymbol{\varphi}^{0}\right)}{\left\|\boldsymbol{\varphi}^{0}\right\|_{X^{\delta}}} \frac{\left\|\boldsymbol{\varphi}^{0}\right\|_{X^{\delta}}}{\|\boldsymbol{\varphi}\|_{X^{\delta}}} \quad$ which implies $\quad \lim _{\delta \rightarrow 0} \sup _{\boldsymbol{\varphi} \in X^{\delta}, \boldsymbol{\varphi} \neq 0} \frac{a_{\delta}\left(\boldsymbol{\psi}_{\delta}^{0}, \boldsymbol{\varphi}\right)}{\|\boldsymbol{\varphi}\|_{X^{\delta}}}=0$.

where we have used (32) and (82) again.

Next, using the result of Proposition 11, we infer from (87) that

$$
\left\|\boldsymbol{\psi}_{\delta}^{0}\right\|_{H^{1 / 2}\left(\Omega_{\alpha \delta}^{ \pm}\right)} \quad \text { is bounded . }
$$

In order to work on a fixed domain, we now again introduce the mappings $F_{ \pm}^{\delta}$ defined in (42) that transforms $\Omega^{ \pm}$into $\Omega_{\alpha \delta}^{ \pm}$. For a vector function $\varphi$ defined on $\Omega_{\alpha \delta}^{ \pm}$we define $\hat{\varphi}$ on $\Omega^{ \pm}$by

$$
\hat{\varphi}(x):=\left[M_{ \pm}^{\delta}\right]^{-1}\left(\varphi \circ F_{ \pm}^{\delta}\right)(x) \quad x \in \Omega^{ \pm} .
$$

This transformation, also known as the $H$ (curl) conforming transform in the finite element literature (see Ref. [29], [18] or [11]) "preserves" the curl operator in the sense that

$$
(\operatorname{curl} \varphi) \circ F_{ \pm}^{\delta}(x)=\left(1-2 \frac{\alpha \delta}{L_{3}}\right)\left[M_{ \pm}^{\delta}\right]^{-1} \operatorname{curl} \hat{\varphi}(x) \quad x \in \Omega^{ \pm}
$$

If $\varphi \in X^{\delta}$ then $\hat{\varphi} \in X^{0}$. We shall equip $X^{0}$ with a $\delta$-dependent norm inspired from the $X_{\delta}$-norm, where we simply take $\alpha=0$ :

$$
\begin{aligned}
\|\hat{\boldsymbol{\psi}}\|_{\hat{X}^{\delta}}^{2} & =\|\hat{\boldsymbol{\psi}}\|_{H\left(\operatorname{curl}, \Omega^{ \pm}\right)}^{2}+\left\|\hat{\boldsymbol{\psi}}_{T}\right\|_{L^{2}\left(\Sigma_{3}^{ \pm}\right)}^{2}+\frac{1}{\delta}\left\|\mathcal{G}_{\omega}\left(\left[e_{3} \times \hat{\boldsymbol{\psi}}\right]\right)\right\|_{H\left(\operatorname{curl}_{\Gamma}, \Gamma\right)}^{2} \\
& +\delta\left\|\left\langle\boldsymbol{\psi}_{T}\right\rangle\right\|_{H\left(\operatorname{curl}_{\Gamma}, \Gamma\right)}^{2} .
\end{aligned}
$$

Again, since the transformation $F_{ \pm}^{\delta}$ goes to identity $(\delta \longrightarrow 0)$, we have if $\boldsymbol{\psi}$ and $\hat{\boldsymbol{\psi}}$ are related by (44):

$$
(1-O(\delta))\|\boldsymbol{\psi}\|_{X^{\delta}}^{2} \leq\|\hat{\boldsymbol{\psi}}\|_{\hat{X}^{\delta}}^{2} \leq(1+O(\delta))\|\boldsymbol{\psi}\|_{X^{\delta}}^{2}
$$


Then we introduce the sesquilinear form $\hat{a}_{\delta}$ defined on $X^{0} \times X^{0}$ by:

$$
\hat{a}_{\delta}(\hat{\boldsymbol{\psi}}, \hat{\boldsymbol{\varphi}}):=a_{\delta}(\boldsymbol{\psi}, \boldsymbol{\varphi})
$$

which gives, thanks to (91)

$$
\begin{aligned}
\hat{a}_{\delta}(\hat{\boldsymbol{\psi}}, \hat{\boldsymbol{\varphi}})= & \left(1-2 \frac{\alpha \delta}{L_{3}}\right) \int_{\Omega^{ \pm}}\left[M_{ \pm}^{\delta}\right]^{-1} \operatorname{curl} \hat{\boldsymbol{\psi}} \cdot\left[M_{ \pm}^{\delta}\right]^{-1} \overline{\operatorname{curl} \hat{\boldsymbol{\varphi}}} d x-i \omega \int_{\Sigma_{3}^{ \pm}} \hat{\boldsymbol{\psi}}_{T} \cdot \overline{\hat{\boldsymbol{\varphi}}_{T}} d s \\
& -\omega^{2}\left(1-2 \frac{\alpha \delta}{L_{3}}\right)^{-1} \int_{\Omega^{ \pm}}\left[M_{ \pm}^{\delta}\right] \hat{\boldsymbol{\psi}} \cdot\left[M_{ \pm}^{\delta}\right] \overline{\hat{\boldsymbol{\varphi}}} d x-\delta \omega^{2} \int_{\Gamma} \mathbf{D}_{m}\left\langle\hat{\boldsymbol{\psi}}_{T}\right\rangle \cdot \overline{\left\langle\hat{\boldsymbol{\varphi}}_{T}\right\rangle} d s \\
& +\delta A_{m} \int_{\Gamma} \operatorname{curl}_{\Gamma}\left\langle\hat{\boldsymbol{\psi}}_{T}\right\rangle \cdot \overline{\operatorname{curl}_{\Gamma}\left\langle\hat{\boldsymbol{\varphi}}_{T}\right\rangle} d s+\frac{\omega^{2}}{\delta}\left\langle\mathcal{G}_{\omega}\left(\left[e_{3} \times \hat{\boldsymbol{\psi}}\right]\right), \overline{\left.\left[e_{3} \times \hat{\boldsymbol{\varphi}}\right]\right\rangle_{\Gamma}} .\right.
\end{aligned}
$$

Using (93), we thus deduce from (87) and (88) that

$$
\lim _{\delta \rightarrow 0}\left\|\hat{\boldsymbol{\psi}}_{\delta}^{0}\right\|_{\hat{X}^{\delta}}=1 \quad \text { and } \quad \lim _{\delta \rightarrow 0} \sup _{\hat{\boldsymbol{\varphi}} \in X^{0}, \boldsymbol{\varphi} \neq 0} \frac{\hat{a}_{\delta}\left(\hat{\boldsymbol{\psi}}_{\delta}^{0}, \hat{\boldsymbol{\varphi}}\right)}{\| \hat{\boldsymbol{\varphi}}_{\hat{X}^{\delta}}}=0
$$

Again, the fact that $F_{ \pm}^{\delta}$ goes to identity as $\delta \rightarrow 0$ implies (this can be proven by interpolation) :

$$
(1-O(\delta))\|\boldsymbol{\psi}\|_{H^{1 / 2}\left(\Omega_{\alpha \delta}^{ \pm}\right)} \leq\|\hat{\boldsymbol{\psi}}\|_{H^{1 / 2}\left(\Omega^{ \pm}\right)} \leq(1+O(\delta))\|\boldsymbol{\psi}\|_{H^{1 / 2}\left(\Omega_{\alpha \delta}^{ \pm}\right)}
$$

Thus, we deduce from (89) that

$$
\hat{\boldsymbol{\psi}}_{\delta}^{0} \text { is bounded in } H^{1 / 2}\left(\Omega^{ \pm}\right) \text {. }
$$

Therefore, one infers the existence of a subsequence, still denoted $\hat{\boldsymbol{\psi}}_{\delta}^{0}$, and of $\hat{\boldsymbol{\psi}} \in$ $H^{1 / 2}\left(\Omega^{ \pm}\right)^{3}$ such that

$$
\hat{\boldsymbol{\psi}}_{\delta}^{0} \rightarrow \hat{\boldsymbol{\psi}}^{ \pm} \quad \text { weakly in } H^{1 / 2}\left(\Omega^{ \pm}\right)^{3} \quad \text { and } \quad \hat{\boldsymbol{\psi}}_{\delta}^{0} \rightarrow \hat{\boldsymbol{\psi}}^{ \pm} \quad \text { strongly in } L^{2}\left(\Omega^{ \pm}\right)^{3} .
$$

From (96) and (92) we also deduce that $\hat{\boldsymbol{\psi}}^{ \pm} \in H\left(\operatorname{curl}, \Omega^{ \pm}\right)$. We next prove that $\hat{\psi}=0$.

Let us denote by $\Omega:=\Omega^{+} \cup \Omega^{-} \cup \Gamma$. We first prove that $\hat{\psi} \in H(\operatorname{curl}, \Omega)$. Indeed, (96) implies that $\mathcal{G}_{\omega}\left[e_{3} \times \hat{\boldsymbol{\psi}}_{\delta}\right]$ strongly converges to 0 in $H\left(\operatorname{curl}_{\Gamma}, \Gamma\right)$. Hence $\mathcal{G}_{\omega}\left[e_{3} \times \hat{\boldsymbol{\psi}}\right]=0$, thus $\left[e_{3} \times \hat{\boldsymbol{\psi}}\right]=0$.

More precisely, we have (the obvious details are left to the reader)

$$
\hat{\boldsymbol{\psi}} \in H_{\mathrm{imp}}(\operatorname{curl}, \Omega):=\left\{\boldsymbol{\varphi} \in H(\operatorname{curl}, \Omega), \boldsymbol{\varphi}_{T} \in L^{2}\left(\Sigma_{3}^{ \pm}\right)^{3}\right\}
$$

and we also deduce from (96) that, $\forall \varphi \in C^{\infty}(\bar{\Omega})$,

$$
\lim _{\delta \rightarrow 0} \delta\left(A_{m} \int_{\Gamma} \operatorname{curl}_{\Gamma}\left\langle\left(\hat{\boldsymbol{\psi}}_{\delta}^{0}\right)_{T}\right\rangle \cdot \operatorname{curl}_{\Gamma} \overline{\left\langle\boldsymbol{\varphi}_{T}\right\rangle}-\omega^{2} \int_{\Gamma} \mathbf{D}_{m}\left\langle\left(\hat{\boldsymbol{\psi}}_{\delta}^{0}\right)_{T}\right\rangle \cdot \overline{\left\langle\boldsymbol{\varphi}_{T}\right\rangle}\right)=0 .
$$

Thus, since, for $\boldsymbol{\varphi} \in C^{\infty}(\bar{\Omega}),\left[e_{3} \times \hat{\boldsymbol{\varphi}}\right]=0$ and $\lim _{\delta \rightarrow 0} \hat{a}_{\delta}\left(\hat{\boldsymbol{\psi}}_{\delta}, \boldsymbol{\varphi}\right)=0$ (by (96)), we deduce from (95) that

$$
\int_{\Omega}\left(\operatorname{curl} \hat{\psi} \cdot \overline{\operatorname{curl} \varphi}-\omega^{2} \hat{\boldsymbol{\psi}} \cdot \bar{\varphi}\right) d x-i \omega \int_{\Sigma_{3}^{ \pm}} \hat{\psi}_{T} \cdot \overline{\varphi_{T}} d s=0, \quad \forall \boldsymbol{\varphi} \in C^{\infty}(\bar{\Omega}) .
$$


Then, by density of $C^{\infty}(\bar{\Omega})$ in $H_{\text {imp }}(\operatorname{curl}, \Omega)$ (see e.g. [7]), we deduce that $\left.\int_{\Omega}\left(\operatorname{curl} \hat{\boldsymbol{\psi}} \cdot \overline{\operatorname{curl} \boldsymbol{\varphi}}-\omega^{2} \hat{\boldsymbol{\psi}} \cdot \overline{\boldsymbol{\varphi}}\right) d x-i \omega \int_{\Sigma_{3}^{ \pm}} \hat{\boldsymbol{\psi}}_{T} \cdot \overline{\boldsymbol{\varphi}_{T}} d s=0, \forall \boldsymbol{\varphi} \in H_{\mathrm{imp}}(\operatorname{curl}, \Omega)\right)$.

The uniqueness result for this variational problem (see for instance Theorem 4.17 in Ref [29]) yields $\hat{\psi}=0$.

We shall derive a contradiction by deducing that $\lim _{\delta \rightarrow 0}\left\|\hat{\boldsymbol{\psi}}_{\delta}^{0}\right\|_{\hat{X}^{\delta}}=0$. First, we just already proved

$$
\lim _{\delta \rightarrow 0}\left\|\hat{\boldsymbol{\psi}}_{\delta}^{0}\right\|_{L^{2}\left(\Omega^{ \pm}\right)^{3}}=0
$$

Using the result of Lemma 15 applied to $\chi \hat{\boldsymbol{\psi}}_{\delta}^{0}$, where $\chi$ is the same cut off function as in the proof of Proposition 11, one concludes that

$$
\chi \hat{\boldsymbol{\psi}}_{\delta}^{0} \text { is bounded in } H^{1}\left(\Omega_{ \pm}\right)
$$

and thus, by trace theorem, that $\left\langle\left(\hat{\boldsymbol{\psi}}_{\delta}^{0}\right)_{T}\right\rangle$ is bounded in $L^{2}(\Gamma)^{3}$. Therefore,

$$
\lim _{\delta \rightarrow 0} \delta\left\|\left\langle\left(\hat{\psi}_{\delta}^{0}\right)_{T}\right\rangle\right\|_{L^{2}(\Gamma)}=0
$$

On the other hand, since $\psi_{\delta}^{0} \in X_{0}^{\delta}$, we easily check that, with obvious notation (we consider traces)

$$
\frac{1}{2}\left(M_{\delta}^{+}\left(\hat{\boldsymbol{\psi}}_{\delta}^{0}\right)^{+}+M_{\delta}^{-}\left(\hat{\boldsymbol{\psi}}_{\delta}^{0}\right)^{+}\right) \cdot e_{3}=\frac{1}{\delta} \operatorname{curl}_{\Gamma} \mathcal{G}_{\omega}\left(\left[e_{3} \times \hat{\boldsymbol{\psi}}_{\delta}^{0}\right]\right)
$$

As, by trace theorem again, $M_{\delta}^{+}\left(\hat{\boldsymbol{\psi}}_{\delta}^{0}\right)^{+}+M_{\delta}^{-}\left(\hat{\boldsymbol{\psi}}_{\delta}^{0}\right)^{+}$is uniformly bounded in $L^{2}(\Gamma)^{3}$, we deduce that

$$
\lim _{\delta \rightarrow 0} \frac{1}{\delta}\left\|\operatorname{curl}_{\Gamma} \mathcal{G}_{\omega}\left(\left[e_{3} \times \hat{\boldsymbol{\psi}}_{\delta}^{0}\right]\right)\right\|_{L^{2}(\Gamma)}^{2}=0
$$

We obtain the desired contradiction by observing that (compare (92) and (95))

$$
\begin{aligned}
\left\|\hat{\boldsymbol{\psi}}_{\delta}^{0}\right\|_{\hat{X}^{\delta}}^{2} \leq C\left(\left|\hat{a}_{\delta}\left(\hat{\boldsymbol{\psi}}_{\delta}^{0}, \hat{\boldsymbol{\psi}}_{\delta}^{0}\right)\right|\right. & +\left\|\hat{\boldsymbol{\psi}}_{\delta}^{0}\right\|_{L^{2}\left(\Omega^{ \pm}\right)^{3}}^{2} \\
& \left.+\delta\left\|\left\langle\hat{\boldsymbol{\psi}}_{\delta}^{0}\right\rangle_{T}\right\|_{L^{2}(\Gamma)^{3}}^{2}+\frac{1}{\delta}\left\|\operatorname{curl}_{\Gamma} \mathcal{G}_{\omega}\left(\left[e_{3} \times \hat{\boldsymbol{\psi}}_{\delta}^{0}\right]\right)\right\|_{L^{2}(\Gamma)}^{2}\right)
\end{aligned}
$$

and that $\lim _{\delta \rightarrow 0} \hat{a}_{\delta}\left(\hat{\boldsymbol{\psi}}_{\delta}^{0}, \hat{\boldsymbol{\psi}}_{\delta}^{0}\right)=0$, by (96).

\section{An application of the stability result}

In this section we shall prove that $\mathbf{E}^{\delta}$, the solution of problem $\mathcal{P}$, approaches $\mathbf{E}_{e}^{\delta}$ (the solution of the exact problem (8)) at second order in $\delta$. To this end, we assume that $\alpha$ is chosen large enough such that $\left(A_{e}, A_{m}, \mathbf{D}_{e}, \mathbf{D}_{m}\right)$ defined by (9) are strictly positive 
so that we can use Proposition 18. Also, we shall assume that the support of the right hand side in does not intersect $\Gamma$ :

$$
\operatorname{supp} f \cap \Gamma=\emptyset
$$

Our proof follows a type of approach that can be found in Ref. [24], [34] and [21]: in a first step, we prove that there exist two functions $\mathbf{E}_{0}$ and $\mathbf{E}_{1}$ defined in $\Omega^{ \pm}$such that

$$
\left\|\mathbf{E}^{\delta}-\mathbf{E}_{0}-\delta \mathbf{E}_{1}\right\|_{H\left(\operatorname{curl}, \Omega_{\alpha \delta}^{ \pm}\right)} \leq C \delta^{2} .
$$

This result is based on an application of Proposition 18 and theorem 17 and on a formal asymptotic expansion of $\mathbf{E}^{\delta}$ with respect to $\delta$. Besides, using the asymptotic expansion of the exact solution $\mathbf{E}_{e}^{\delta}$ in $\delta$, using for instance matched asmptotics as in Ref. [17] (see for instance also Ref. [35],[22] and [25] for general results on this topic), we can also prove an optimal estimate of $\mathbf{E}^{\delta}-\left(\mathbf{E}_{0}+\delta \mathbf{E}_{1}\right)$, that is valid in any (fixed) domain that does not intersect a small neighbourhood of $\Gamma$, namely in any $(\gamma>0$ being given, possibly arbitrarily small)

$$
\Omega_{\gamma}:=\left\{\left(x_{1}, x_{2}, x_{3}\right) \in \mathbb{R}^{3} /\left|x_{1}\right|<L_{1} / 2,\left|x_{2}\right|<L_{2} / 2 \text { and } \gamma<\left|x_{3}\right|<L_{3} / 2\right\} .
$$

Such estimates have been established in [15]. Combining the previous two estimates, we obtain an estimate of $\mathbf{E}_{e}^{\delta}-\mathbf{E}^{\delta}$ in $\Omega_{\gamma}$. The precise result is the following.

Proposition 19. Assume that (6), (7), (99) and (100) hold. Assume that the frequency $\omega$ satisfies the hypothesis (6). Then, for any $0<\gamma<L_{3} / 2$, there exist a constant $\delta_{\gamma}>0$ and a positive constant $C_{\gamma}$, such that

$$
\forall \delta<\delta_{\gamma}, \quad\left\|\mathbf{E}_{e}^{\delta}-\mathbf{E}^{\delta}\right\|_{H\left(\operatorname{curl}, \Omega_{\gamma}\right)} \leq C_{\gamma} \delta^{2} .
$$

The remaining of this section in dedicated to the proof (in three steps) of this proposition.

\section{Step 1. Formal asymptotic expansion of $\mathrm{E}^{\delta}$.}

We look for a power series expansion of $\mathbf{E}^{\delta}$ with respect to $\delta$ of the form

$$
\mathbf{E}^{\delta}=\sum_{n \in \mathbb{N}} \delta^{n} \mathbf{E}_{n}
$$

where the fields $\mathbf{E}_{n}$ are searched functions in $\Omega^{+} \cup \Omega^{-}$which are smooth respectively in $\overline{\Omega^{+}}$and $\overline{\Omega^{-}}$. Inserting the previous expansion in problem $\mathcal{P}$, and (formally) separating the different powers of $\delta$, it is easily seen that the fields $\mathbf{E}_{n}$ satisfy the Maxwell equations in $\Omega^{ \pm}$

$$
\text { curl curl } \mathbf{E}_{n}-\omega^{2} \mathbf{E}_{n}= \begin{cases}f & \text { if } n=0 \\ 0 & \text { otherwise }\end{cases}
$$

as well as the boundary conditions:

$$
\left\{\begin{array}{l} 
\pm e_{3} \times \operatorname{curl} \mathbf{E}_{n}=-i \omega\left(\mathbf{E}_{n}\right)_{T} \text { on } \Sigma_{3}^{ \pm} \\
\mathbf{E}_{n} \times\left. e_{i}\right|_{\Sigma_{i}^{+}}=\mathbf{E}_{n} \times\left. e_{i}\right|_{\Sigma_{i}^{-}}, \text {and } \operatorname{curl} \mathbf{E}_{n} \times\left. e_{i}\right|_{\Sigma_{i}^{+}}=\operatorname{curl} \mathbf{E}_{n} \times\left. e_{i}\right|_{\Sigma_{i}^{-}}
\end{array}\right.
$$


Deriving transmission conditions for the $E_{j}^{\prime} s$ from the transmission conditions (5) is more involved. Let us give the idea of the formal computations. To exploit (102), we have to evaluate the traces of $e_{3} \times \mathbf{E}_{j}$ or $e_{3} \times \operatorname{curl} \mathbf{E}_{j}$ on $\Gamma_{\alpha \delta}$. Using (formally) Taylor expansions with respect to the $x_{3}$ variable, we get an expansion of such traces in powers of $\delta$ which are expressed only in terms of tangential traces of $e_{3} \times \mathbf{E}_{j}$ or $e_{3} \times \operatorname{curl} \mathbf{E}_{j}$ on $\Gamma$ thanks to the fact $x_{3}$ derivatives are eliminated by using the interior Maxwell's equations and replaced by tangential derivatives. As a result, the transmission conditions are posed on the interface $\Gamma$. For the sake of conciseness, we do not develop the calculations and we restrict ourselves to present the resulting jump conditions (see Ref. [15] for more details).

First, the limit field $\mathbf{E}_{0}$ satisfies the following homogeneous jump conditions on $\Gamma$,

$$
\left[e_{3} \times \mathbf{E}_{0}\right]=0, \quad \text { and } \quad\left[e_{3} \times \operatorname{curl} \mathbf{E}_{0}\right]=0,
$$

which simply expresses the fact that $E_{0}$ "does not see" the rough interface. On the other hand, for $j=1$ or $j=2$, the jump conditions for $\mathbf{E}_{j}$ are recursively given by

$$
\left\{\begin{array}{l}
{\left[e_{3} \times \mathbf{E}_{j}\right]+\frac{\left(A_{e}-2 \alpha\right)}{\omega^{2}} \operatorname{curl}_{\Gamma} \operatorname{curl}_{\Gamma}\left\langle\left(\operatorname{curl} \mathbf{E}_{j-1}\right)_{T}\right\rangle-\left(\mathbf{D}_{e}-2 \alpha\right)\left\langle\left(\operatorname{curl} \mathbf{E}_{j-1}\right)_{T}\right\rangle=0,} \\
{\left[e_{3} \times \operatorname{curl} \mathbf{E}_{j}\right]+\left(A_{m}-2 \alpha\right) \operatorname{curl}_{\Gamma} \overrightarrow{c u r l}_{\Gamma}\left\langle\left(\mathbf{E}_{j-1}\right)_{T}\right\rangle-\omega^{2}\left(\mathbf{D}_{m}-2 \alpha\right)\left\langle\left(\mathbf{E}_{j-1}\right)_{T}\right\rangle=0 .}
\end{array}\right.
$$

The existence of $E_{j}$ 's for $j=0,1,2$ results from standard results from Maxwell equations. Note that the $C^{\infty}$ regularity of each $E_{j}^{+}$and $E_{j}^{-}$in a neighborhood of $\Gamma$ is guaranteed by the fact that the support of the source term $f$ does not intersect $\Gamma$.

\section{Step 3. Justification of the asymptotic expansion of $\mathrm{E}^{\delta}$.}

We now consider the error $e_{2}^{\delta}$ between $\mathbf{E}^{\delta}$ (the solution of $\mathcal{P}$ ) and its truncated expansion at second order:

$$
e_{2}^{\delta}:=\mathbf{E}^{\delta}-\left(\mathbf{E}_{0}+\delta \mathbf{E}_{1}+\delta^{2} \mathbf{E}_{2}\right)
$$

Let $\mathcal{D}_{\alpha, \delta}$ and $\mathcal{N}_{\alpha, \delta}$ be the generalized trace operators defined by ( $u$ denotes a smooth enough vector field):

$$
\left\{\begin{array}{l}
\mathcal{D}_{\alpha, \delta} u:=\left[e_{3} \times u\right]_{\alpha}+\delta\left(\frac{A_{e}}{\omega^{2}} \operatorname{curl}_{\Gamma} \operatorname{curl}_{\Gamma}\left(\langle\operatorname{curl} u\rangle_{\alpha}\right)_{T}-\mathbf{D}_{e}\left(\langle\operatorname{curl} u\rangle_{\alpha}\right)_{T}\right) \\
\mathcal{N}_{\alpha, \delta} u:=\left[e_{3} \times \operatorname{curl} u\right]_{\alpha}+\delta\left(A_{m} \operatorname{curl}_{\Gamma} \operatorname{curl}_{\Gamma}\left\langle(u)_{T}\right\rangle_{\alpha}-\omega^{2} \mathbf{D}_{m}\left\langle u_{T}\right\rangle_{\alpha}\right)
\end{array}\right.
$$

Using Taylor expansion, it is easily seen that $e_{2}^{\delta}$ satisfies a problem of the form

$$
\begin{cases}\operatorname{curl} \operatorname{curl} e_{2}^{\delta}-\omega^{2} e_{2}^{\delta}=0 & \text { in } \Omega_{\alpha \delta}^{+} \cup \Omega_{\alpha \delta}^{-}, \\ \mathcal{D}_{\alpha, \delta} e_{2}^{\delta}=\delta^{3} h^{\delta}, \quad \mathcal{N}_{\alpha, \delta} e_{2}^{\delta}=\delta^{3} g^{\delta}, & \text { on } \Gamma \\ \pm e_{3} \times \operatorname{curl} e_{2}^{\delta}=-i \omega\left(e_{2}^{\delta}\right)_{T}, & \text { on } \Sigma_{3}^{ \pm}, \\ e_{2}^{\delta} \times\left. e_{i}\right|_{\Sigma_{i, \delta}^{+}}=e_{2}^{\delta} \times\left. e_{i}\right|_{\Sigma_{i, \delta}^{-}}, \text {and } \operatorname{curl} e_{2}^{\delta} \times\left. e_{i}\right|_{\Sigma_{i, \delta}^{+}}=\operatorname{curl} e_{2}^{\delta} \times\left. e_{i}\right|_{\Sigma_{i, \delta}^{-},} & \end{cases}
$$


where the terms $\delta^{3} g^{\delta}$ and $\delta^{3} h^{\delta}$ come from the evaluation of $\mathcal{D}_{\alpha, \delta}$ and $\mathcal{N}_{\alpha, \delta}$ on $\mathbf{E}_{0}^{ \pm}+$ $\delta \mathbf{E}_{1}^{ \pm}+\delta^{2} \mathbf{E}_{2}^{ \pm}$. To exploit the jump conditions (105), we need to relate these traces with their equivalent on $\Gamma$. This can be done using Taylor expansions in the $x_{3}$ variable with integral rest. Doing so, we make appear automatically the $\delta^{3}$ factor and it is not difficult (although tedious) to establish that $g^{\delta}$ and $h^{\delta}$ (that are expressed in terms of integrals over $\left.\pm x_{3} \in\right] 0, \alpha \delta\left[\right.$ of functions involving $\mathbf{E}_{0}, \mathbf{E}_{1}, \mathbf{E}_{2}$ and some of their derivatives, up to third order) satisfy the uniform estimates (we use standard elliptic regularity results for $\mathbf{E}_{0}, \mathbf{E}_{1}, \mathbf{E}_{2}$ and the assumption (100) for the support of $f$.)

$$
\left\|g^{\delta}\right\|_{H^{-1 / 2}\left(\operatorname{div}_{\Gamma}, \Gamma\right)}+\left\|h^{\delta}\right\|_{L^{2}(\Gamma)} \leq C\|f\|_{L^{2}(\Omega)} .
$$

Then, note that $e_{2}^{\delta}$ belongs to $X^{\delta}$ (but it does not belong to $X_{0}^{\delta}$ ). Writing the previous problem in a variational form, we get

$$
\forall \boldsymbol{\varphi} \in X^{\delta}, \quad a_{\delta}\left(e_{2}^{\delta}, \boldsymbol{\varphi}\right)=\left\langle L^{\delta}, \boldsymbol{\varphi}\right\rangle
$$

where the linear form in $L^{\delta} \in\left(X^{\delta}\right)^{\prime}$ is given by

$$
\forall \boldsymbol{\varphi} \in X^{\delta}, \quad\left\langle L^{\delta}, \boldsymbol{\varphi}\right\rangle:=\delta^{2} \omega^{2}\left\langle\mathcal{G}_{\omega}\left(g^{\delta}\right),\left[e_{3} \times \varphi\right]_{\alpha}\right\rangle+\delta^{3} \int_{\Gamma} h^{\delta} \cdot \overline{\langle\varphi\rangle_{\alpha}} .
$$

Thanks to the stability result of Proposition 18, it is clear that the estimate of will be driven by the estimation of the norm of $L^{\delta} \in\left(X^{\delta}\right)^{\prime}$. For this we observe that, using in particular the symmetry of $\mathcal{G}_{\omega}$ (see (14))

$$
\begin{aligned}
\left|\left\langle\mathcal{G}_{\omega}\left(g^{\delta}\right),\left[e_{3} \times \varphi\right]_{\alpha}\right\rangle\right| & \leq C\left\|g^{\delta}\right\|_{H^{-1 / 2}\left(\operatorname{div}_{\Gamma}, \Gamma\right)}\left\|\mathcal{G}_{\omega}\left(\left[e_{3} \times \varphi\right]_{\alpha}\right)\right\|_{H^{-1 / 2}\left(\operatorname{curl}_{\Gamma}, \Gamma\right)} \\
& \leq C \delta^{\frac{1}{2}}\left\|g^{\delta}\right\|_{H^{-1 / 2}\left(\operatorname{div}_{\Gamma}, \Gamma\right)}\left\|\delta^{-\frac{1}{2}} \mathcal{G}_{\omega}\left(\left[e_{3} \times \varphi\right]_{\alpha}\right)\right\|_{H\left(\operatorname{curl}_{\Gamma}, \Gamma\right)} .
\end{aligned}
$$

while on the other hand we have

$$
\left|\int_{\Gamma} h \cdot \overline{\langle\boldsymbol{\varphi}\rangle_{\alpha}}\right| \leq \delta^{-\frac{1}{2}}\left\|h^{\delta}\right\|_{L^{2}(\Gamma)}\left\|\delta^{\frac{1}{2}} \overline{\langle\varphi\rangle_{\alpha}}\right\|_{L^{2}(\Gamma)} .
$$

Combining (107), (109), (110) and (111), we obtain, thanks tothe definition of the $X^{\delta}$-norm (27)

$$
\forall \varphi \in X^{\delta}, \quad\left|\left\langle L^{\delta}, \varphi\right\rangle\right| \leq C \delta^{\frac{5}{2}}\|f\|_{L^{2}(\Omega)}\|\varphi\|_{X^{\delta}} .
$$

Applying theorem 17 and proposition 18, for any $\delta$ small enough, we get

$$
\left\|e_{2}^{\delta}\right\|_{X^{\delta}}=\left\|\mathbf{E}^{\delta}-\left(\mathbf{E}_{0}+\delta \mathbf{E}_{1}+\delta^{2} \mathbf{E}_{2}\right)\right\|_{X^{\delta}} \leq C \delta^{5 / 2}\|f\|_{L^{2}(\Omega)} .
$$

Let us introduce a constant $\gamma$ such that $0<\gamma<L_{3} / 2$ and consider the domain $\Omega_{\gamma}$

$$
\Omega_{\gamma}:=\left\{\left(x_{1}, x_{2}, x_{3}\right) \in \mathbb{R}^{3}\left|x_{1}\right|<L_{1} / 2,\left|x_{2}\right|<L_{2} / 2 \text { and } \gamma<\left|x_{3}\right|<L_{3} / 2\right\}
$$

Finally, from the previous estimate and the triangular inequality, it is easily seen that there exist two positive constants $\delta_{0}$ and $C$ such that

$$
\forall \delta<\delta_{0}, \quad\left\|\mathbf{E}^{\delta}-\left(\mathbf{E}_{0}+\delta \mathbf{E}_{1}\right)\right\|_{H\left(\operatorname{curl}, \Omega_{\gamma}\right)} \leq C \delta^{2} .
$$




\section{Step 3. Derivation of the error estimates.}

The asymptotic expansion of the exact solution $\mathbf{E}_{e}^{\delta}$ is much more involved than the approximate one: we have to take into account both the thickness and the periodicity of the thin layer. For instance, we can construct this asymptotics using a method that mixes matched asymptotic expansions and homogenization techniques (see for instance Ref. [33, 2, 28]). This is in particular where the symmetry assumption (7) plays a role. All this has been done in [15] or [16] from which we extract the useful result for our purpose (note that this is where the restriction to the domain $\Omega_{\gamma}$ appears), namely following estimate:

$\forall \gamma>0, \exists \delta_{0}>0$ and $\exists C>0$ such that, $\forall \delta<\delta_{0}, \quad\left\|\mathbf{E}_{e}^{\delta}-\left(\mathbf{E}_{0}+\delta \mathbf{E}_{1}\right)\right\|_{H\left(\operatorname{curl}, \Omega_{\gamma}\right)} \leq C \delta^{2}$. (114)

From inequalities (114) and (113), we immediately deduce the estimate (101), which concludes the proof of Proposition 19.

\section{References}

[1] T. Abboud and H. Ammari. Diffraction at a curved grating: TM and TE cases, homogenization. J. Math. Anal. Appl., 202(3):995-1026, 1996.

[2] Y. Achdou. Etude de la réflexion d'une onde électromagnétique par un métal recouvert d'un revêtement métallisé. Technical report, INRIA, 1989.

[3] Y ACHDOU. Effect of a thin metallized coating on the reflection of and electromagnetic wave. Comptes rendus de l'académie des sciences série Imathématique, 314(3):217-222, JAN 301992.

[4] A. Alonso and A. Valli. Some remarks on the characterization of the space of tangential traces of $H(\operatorname{rot} ; \Omega)$ and the construction of an extension operator. Manuscripta Math., 89(2):159-178, 1996.

[5] C. Amrouche, C. Bernardi, M. Dauge, and V. Girault. Vector potentials in threedimensional non-smooth domains. Math. Methods Appl. Sci., 21(9):823-864, 1998.

[6] B. Aslanyurek, Houssem Haddar, and H. Shahinturk. Generalized impedance boundary conditions for thin dielectric coatings with variable thickness. Wave Motion, 48(7):681-700, November 2011.

[7] F. Ben Belgacem, C. Bernardi, M. Costabel, and M. Dauge. Un résultat de densité pour les équations de Maxwell. C. R. Acad. Sci. Paris Sér. I Math., 324(6):731736, 1997.

[8] M. Sh. Birman and M. Z. Solomyak. $L_{2}$-theory of the Maxwell operator in arbitrary domains. Uspekhi Mat. Nauk, 42(6(258)):61-76, 247, 1987.

[9] A. Buffa, M. Costabel, and D. Sheen. On traces for $\mathbf{H}(\mathbf{c u r l}, \Omega)$ in Lipschitz domains. J. Math. Anal. Appl., 276(2):845-867, 2002.

[10] S. Chun, H. Haddar, and J. S. Hesthaven. High-order accurate thin layer approximations for time-domain electromagnetics, Part II: transmission layers. $J$. Comput. Appl. Math., 234(8):2587-2608, 2010. 
[11] G. Cohen. Higher-order numerical methods for transient wave equations. Scientific Computation. Springer-Verlag, Berlin, 2002. With a foreword by R. Glowinski.

[12] M. Costabel. A remark on the regularity of solutions of Maxwell's equations on Lipschitz domains. Math. Methods Appl. Sci., 12(4):365-368, 1990.

[13] M. Costabel and M. Dauge. Singularities of electromagnetic fields in polyhedral domains. Arch. Ration. Mech. Anal., 151(3):221-276, 2000.

[14] M. Costabel, M. Dauge, and S. Nicaise. Singularities of Maxwell interface problems. M2AN Math. Model. Numer. Anal., 33(3):627-649, 1999.

[15] B. Delourme. Modèles et asymptotiques des interfaces fines et périodiques en électromagnetisme. PhD thesis, Université Paris 6, 2010.

[16] B. Delourme. High order asymptotics for the scattering of electromagnetic waves from thin periodic layers, 2012.

[17] B. Delourme, H. Haddar, and P. Joly. Approximate models for wave propagation across thin periodic interfaces, 2011.

[18] F. Dubois. Du tourbillon au champ de vitesse, lectures notes. Technical report, 2000.

[19] V. Girault and P.A. Raviart. Finite element methods for Navier-Stokes equations, volume 5 of Springer Series in Computational Mathematics. Springer-Verlag, Berlin, 1986. Theory and algorithms.

[20] H. Haddar and P. Joly. Stability of thin layer approximation of electromagnetic waves scattering by linear and nonlinear coatings. J. Comput. Appl. Math., 143(2):201-236, 2002.

[21] H. Haddar, P. Joly, and H.M. Nguyen. Generalized impedance boundary conditions for scattering problems from strongly absorbing obstacles: the case of Maxwell's equations. Math. Models Methods Appl. Sci., 18(10):1787-1827, 2008.

[22] A. M. Il'in. Matching of asymptotic expansions of solutions of boundary value problems, volume 102 of Translations of Mathematical Monographs. American Mathematical Society, Providence, RI, 1992. Translated from the Russian by V. Minachin [V. V. Minakhin].

[23] P. Joly, C. Poirier, J. E. Roberts, and P. Trouve. A new nonconforming finite element method for the computation of electromagnetic guided waves. I. Mathematical analysis. SIAM J. Numer. Anal., 33(4):1494-1525, 1996.

[24] P. Joly and A. Semin. Construction and analysis of improved Kirchoff conditions for acoustic wave propagation in a junction of thin slots. In Paris-Sud Working Group on Modelling and Scientific Computing 2007-2008, volume 25 of ESAIM Proc., pages 44-67. EDP Sci., Les Ulis, 2008.

[25] P. Joly and S. Tordeux. Matching of asymptotic expansions for wave propagation in media with thin slots. I. The asymptotic expansion. Multiscale Model. Simul., 5(1):304-336 (electronic), 2006. 
[26] A. Kirsch and P. Monk. A finite element/spectral method for approximating the time-harmonic Maxwell system in $\mathbf{R}^{3}$. SIAM J. Appl. Math., 55(5):1324-1344, 1995.

[27] A. Kirsch and P. Monk. Corrigendum: "A finite element/spectral method for

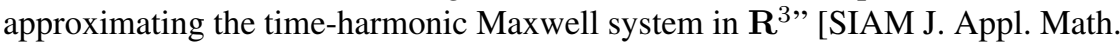
55 (1995), no. 5, 1324-1344; MR1349312 (96j:65124)]. SIAM J. Appl. Math., 58(6):2024-2028 (electronic), 1998.

[28] A.L. Madureira and F. Valentin. Asymptotics of the Poisson problem in domains with curved rough boundaries. SIAM J. Math. Anal., 38(5):1450-1473 (electronic), 2006/07.

[29] P. Monk. Finite element methods for Maxwell's equations. Numerical Mathematics and Scientific Computation. Oxford University Press, New York, 2003.

[30] J.C. Nédélec. Acoustic and electromagnetic equations, volume 144 of Applied Mathematical Sciences. Springer-Verlag, New York, 2001. Integral representations for harmonic problems.

[31] C. Poirier. Guides d'ondes électromagnétiques : analyse mathématique et numérique. $\mathrm{PhD}$ thesis, Université de Nantes, 1994.

[32] J.R. Poirier, A. Bendali, and P. Borderies. Impedance boundary conditions for the scattering of time-harmonic waves by rapidly varying surfaces. Antennas and Propagation, IEEE Transactions on, 54(3):995-1005, 2006.

[33] E. Sánchez-Palencia. Un problème d' écoulement lent d'une fluide visqueux incompressible au travers d'une paroi finement perforée. Technical report, Electricité de France, 1985.

[34] K. Schmidt. High-order numerical modelling of highly conductive thin sheets. $\mathrm{PhD}$ thesis, ETH Zurich, 2008.

[35] M. Van Dyke. Perturbation methods in fluid mechanics. Applied Mathematics and Mechanics, Vol. 8. Academic Press, New York, 1964.

[36] Ch. Weber. A local compactness theorem for Maxwell's equations. Math. Methods Appl. Sci., 2(1):12-25, 1980.

[37] A. Zebic. Conditions de frontière équivalentes en électromagnétisme. $\mathrm{PhD}$ thesis, Université Paris 6, 1994. 